\title{
The pharmacodynamics of antibiotic treatment $\dagger$
}

\author{
IMRAN MUDASSAR and HAL SMITH* \\ Department of Mathematics and Statistics, Arizona State University, Tempe, AZ, 85287, USA \\ (Received 22 June 2006; in final form 31 October 2006)
}

\begin{abstract}
We derive models of the effects of periodic, discrete dosing or constant dosing of antibiotics on a bacterial population whose growth is checked by nutrient-limitation and possibly by host defenses. Mathematically rigorous results providing sufficient conditions for treatment success, i.e. the elimination of the bacteria, as well as for treatment failure, are obtained. Our models can exhibit bi-stability where the infection-free state and an infection-state are locally stable when antibiotic dosing is marginal. In this case, treatment success may occur only for sub-threshold level infections.
\end{abstract}

Keywords: Pharmacokinetics; Pharmacodynamics; Antibiotic; Infection; Plasmid

\section{Introduction}

The pharmacology of antibiotics (or any drug) can be divided into pharmacokinetics and pharmacodynamics. Pharmacokinetics describes the movement of antibiotic into, through and out of the body whereas pharmacodynamics describes the relationship between the concentration of antibiotic, its effect on target bacteria (growth or decay) and factors influencing this relationship [7]. The elimination of drug either by metabolism or excretion is very important since it determines the dosing frequency [1]. One of the important objectives of pharmacokinetics is to decide the optimal dosing frequency of an antibiotic for successful treatment. On the other hand pharmacodynamics describes in detail the relationship between drug concentration and its effects on the bacterial population in order to achieve the maximum removal of bacteria from the host.

Mathematical modeling of the effects of drug treatment has long been used side-by-side with experimental studies $[1,3-5,25,27,38]$. However, most mathematical models of the effects of antimicrobial agents on bacterial populations assume that bacteria grow at an exponential rate in the absence of the antimicrobial agent. In that case, one need only determine the pharmacodynamic function for the agent, that is, a mathematical expression for the decline in growth rate resulting from a given concentration of the antimicrobial agent. Once this has been achieved, then the minimum inhibitory concentration (MIC) for the agent is determined as that concentration of the agent at which the growth rate, $\psi=\psi(A)$ vanishes, where $A$ denotes the antibiotic concentration. One can then explore the response of bacteria to various dosing strategies. The expected outcome of this approach is that a dosing strategy

\footnotetext{
*Corresponding author. Email: halsmith@math.la.asu.edu

$\dagger$ Supported by NSF Grant DMS 0107160.

Computational and Mathematical Methods in Medicine ISSN 1748-670X print/ISSN 1748-6718 online () 2006 Taylor \& Francis

http://www.tandf.co.uk/journals

DOI: $10.1080 / 10273660601122773$
} 
that is insufficient to control the population results in its exponential growth while an adequate dosing drives the population to extinction.

It is often found that fast growing bacteria are more susceptible to antibiotic treatment as compared to slow growing ones $[3,6,22,26,28,36,37]$. A slow growth rate due to the restricted availability of nutrients could be a major contributor toward insensitivity of bacteria to antibiotic. Therefore it is plausible that in some cases the pharmacodynamic function should depend on both the antimicrobial agent and limiting nutrient levels, i.e. $\psi=\psi(S, A)$ where $S$ denotes limiting resource level. It has been noted that bacteria multiply more slowly in an experimental animal than in vitro [4,6], suggesting nutrient limitation in vivo. Corpet et al. [4] introduce pharmacodynamic functions which depend on limiting both nutrient and antimicrobial agent. Cogan [3] does as well in his considerations of persister cells. Cozens et al. [6] note that the restricted availability of iron and other nutrients appears to be typical of infection states. Roberts and Stewart [28] construct a mathematical model to explore the possibility that the observed antibiotic tolerance of biofilms is due in part to nutrient limitation reducing bacterial growth and hence killing rates. Even if resource supply rates are relatively constant, one expects significant depletion in local resource levels as a bacterial infection progresses and we expect these changes to play a role in treatment by antimicrobial agents.

We derive simple models of the effects of periodically-administered discrete dosing or constant antimicrobial dosing strategies on a bacterial population whose growth is checked by nutrient-limitation and possibly by host defenses if not by the antimicrobial agent itself. Distinguishing features of our model are the inclusion of nutrient limitation of microbial growth and accounting for the removal of antimicrobial agent by association with bacteria. Earlier models such as Austin et al. [1] mention nutrient limitation but employ a logistic bacterial growth rate rather than explicitly treating nutrient limitation. Like the model of Austin et al. ours follows the pre-treatment infection dynamics as well as the post-treatment course of bacterial levels.

Most models of antibiotic treatment, e.g. Ref. [1], assume that the pathogen has no effect on antimicrobial concentration; its concentration at the site of infection is simply an input to the model. In our model, the antibiotic concentration at the site of infection is a dynamic variable with the periodic dosing as input. Standard pharmacokinetics are used but we include a bacteria-dependent drug removal rate, attributable to the association of the drug with bacterial cells, which may be important in some cases.

Mathematically rigorous results are obtained for our models that provide sufficient conditions for treatment success - the elimination of the bacteria - as well as for treatment failure. In the case that antibiotic concentration is assumed to be oscillatory, the MIC, which may depend on resource levels, is no longer the critical parameter. Rather the key parameter is an "invasion eigenvalue" which determines whether bacteria can invade an environment in which antibiotic levels have attained their asymptotic periodic pharmacokinetic regime $A^{*}(t)$ : typically, a repeating cycle of exponential decay and recovery following a discrete dose. (See figure 1.) We refer to this regime as the APPR for simplicity. The "invasion eigenvalue", in fact a Floquet exponent, is the average growth rate over a dose-cycle $T$ :

$$
\lambda=\frac{1}{T} \int_{0}^{T} \psi\left(S^{0}, A^{*}(t)\right) \mathrm{d} t
$$

where $S^{0}$ is the resource level in the absence of bacteria and $A^{*}(t)=A^{*}(t+T)$ is the APPR. Although it is often remarked that the amount of time during which $A^{*}(t)$ exceeds the MIC is 

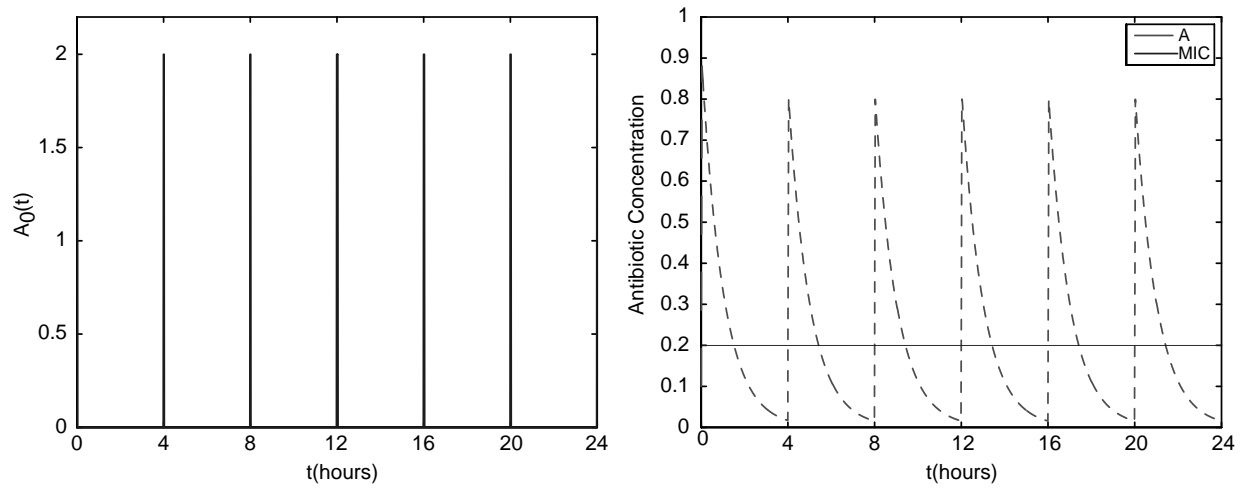

Figure 1. The left figure depicts periodic discrete dosing of an antibiotic. The resulting pharmacokinetics $A^{*}(t)$ and the MIC level of $A$ at which $\lambda=0$ appears on the right.

critical, $\lambda$ is a much more subtle parameter. The generally nonlinear dependence of $\psi$ on $A$, means that $\lambda$ can differ substantially from the growth rate evaluated at the average dose level

$$
\lambda \neq \psi\left(S^{0},\left[A^{*}\right]_{m}\right), \quad\left[A^{*}\right]_{m}=\frac{1}{T} \int_{0}^{T} A^{*}(t) \mathrm{d} t
$$

In vivo, bacteria usually become established before intervention in the form of antibiotic treatment is administered, rather than the other way around where a small inoculum of bacteria is introduced to a pre-established APPR. Therefore, one may be sceptical that the invasion eigenvalue $\lambda$ has relevance for treatment. However, in order to eliminate bacteria one must drive down the bacterial population to near extinction where the premise of the invasion eigenvalue approach is satisfied because the state vector approximates the APPR. This suggests and we prove, that when $\lambda>0$, then bacteria persist and treatment fails because the bacteria-free state is unstable. This is expected since, on average over a dosing cycle, bacteria introduced into the APPR grow when rare. However, $\lambda<0$ implies only local stability of the bacteria-free state. This means that an indeterminately small bacterial population introduced into the APPR is eliminated but one cannot conclude that a larger inoculum is necessarily eliminated. While there are important cases when $\lambda<0$ implies treatment success regardless of initial conditions, in general it does not. In particular, the bacteria-free state can be locally attracting, guaranteeing successful treatment for very small initial bacterial populations and yet a larger initial population may lead to a stable endemic infection state. We find such bistable dynamics in our model when the antimicrobial dosing is marginal. This dynamic feature is possible when the removal rate of antibiotic depends significantly on bacterial density. In this case, provided sufficient nutrient is present and antibiotic dosing is marginal, high bacterial density can significantly reduce antibiotic concentration thereby reducing its effect. The potential for bistability in antibiotic treatment appears not to have been observed in previous mathematical modeling. It suggests the obvious-early treatment of infection by antibiotics, before bacterial populations have reached high levels, is optimal. The larger message is that antibiotic dosing levels may need to take account of nutrient availability.

Because of the failure of the inequality $\lambda<0$ to guarantee treatment success, we are motivated to find stronger threshold inequalities of the form $\lambda_{*}<0$ where $\lambda<\lambda_{*}$ which do guarantee treatment success. We are successful in identifying thresholds $\lambda_{*}$ able to be 
expressed entirely in terms of the mean dosing strength, the pharmacodynamic function and removal rates of antibiotic, resource and bacteria.

The choice of pharmacodynamic function $\psi$ is largely an educated guess even in the case when it can be expected to depend only on antimicrobial agent concentration $(\psi=\psi(A))$ [1]. The so-called $E_{\max }$ model, based on drug-receptor interaction, is rather standard [24,15]. In general, a pharmacodynamic function will depend on the particular antimicrobial agent as well as the organism. There appears to be no well-developed pharmacodynamic theory which includes both nutrient and antibiotic. Undoubtedly, there are many possibilities depending on whether or not the two act independently or not. Cogan [3] introduces a pharmacodynamic function depending on the product of a function of nutrient and a function of antibiotic. Corpet et al. [4] modify the classical Monod growth rate by assuming either the maximum growth rate or the half-saturation constant is influenced by antibiotic concentration. As these functions are based on educated hunches, our modeling study can only be qualitative in nature exploring possible behavior given only qualitative information about the pharmacodynamic function.

It is well-known that bacterial populations are heterogeneous in their susceptibility to antimicrobial agents $[3,15,25]$. Furthermore, many human pathogens have acquired resistance to the antibiotic of choice which has had an important impact on public health. We also formulate a model where the bacterial population is heterogeneous in its response to the antimicrobial agent either due to mutation or the presence or absence of a transmissible plasmid encoding resistance to the antimicrobial agent. Successful treatment requires eradication of both resistant and susceptible populations. This model has potentially two types of disease states: one with only the susceptible population present and one with both susceptible and resistant populations. We are able to give sufficient conditions for successful treatment and sufficient conditions for treatment failure, i.e. the persistence of a bacterial population. In this case, there are two distinct values of $\lambda$ : $\lambda_{\mathrm{S}}$ for susceptible and $\lambda_{\mathrm{r}}$ for the resistant population. It is important to understand the conditions favoring the establishment of the mixed population of resistant and susceptible types.

Our work appears to be the first to attempt to obtain mathematically rigorous, explicit sufficient conditions for treatment success and for treatment failure in simple, but hopefully not too simple, mathematical models of periodic drug dosing in vitro and in vivo. Our models combine both the pharmacokinetics of the antibiotic and the pharmacodynamics of its effect on a bacterial population in order to study antibiotic treatment. The mathematical theory of persistence plays a major role here as it is used to characterize treatment failure.

In the next section, we formulate and analyze models of antibiotic treatment of a bacterial population which is homogeneous in its response to the antimicrobial agent. In the following section, models containing a susceptible and resistant population are developed. Proofs of our results are relegated to an appendix.

\section{Model of antibiotic treatment}

We first assume an in vitro environment that can be modeled as a chemostat with the added feature that an antibiotic is also input from the external source. Later, we will introduce a more general model suitable for in vivo environments. Let $S$ denote the concentration of nutrient sustaining microbial growth, $u$ denote the density of bacteria, and $A$ denote the concentration of an antibiotic. If we view the antibiotic as bactericidal that is as killing 
bacteria, then the equations take the form

$$
\begin{aligned}
S^{\prime} & =D\left(S^{0}-S\right)-\gamma^{-1} f(S) u \\
A^{\prime} & =D\left(A_{0}(t)-A\right)-u p(A) \\
u^{\prime} & =[f(S)-D-g(S, A)] u
\end{aligned}
$$

We assume fresh nutrient at constant concentration $S^{0}$ is input and $A_{0}(t)$ is the concentration of the antibiotic at time $t$ in the input. Parameter $\gamma$ is the yield constant and $f(S)$ is the growth rate of bacteria at nutrient concentration $S$. Typically, we take $f$ to be Monod type but our results hold more generally. We require only that the growth (or uptake) function $f(S)$ be monotone increasing in $S$ :

$$
f(0)=0, \quad f^{\prime}(S) \geq 0
$$

$D$ is the dilution rate.

Typically, antibiotics are administered in either a constant dose $A_{0}(t) \equiv A_{0}=$ constant or periodically $A_{0}(t)=A_{0}(t+T) \geq 0$ with dosing period $T$. While our model allows a general non-negative periodic dosing function $A_{0}(t)$, in practice it is typically a sequence of discrete doses which might be approximated by:

$$
A_{0}(t)=\sum_{i} d \delta(t-i T)
$$

Parameter $d$ measures the dose and $\delta$ is the Dirac impulse function.

Function $g=g(S, A)$ is the so-called pharmacodynamic function which describes the kill rate or alternatively, the reduction in the growth rate, induced by the antibiotic agent per unit of bacteria. In general, the killing rate depends on the bacteria and the antibiotic used as well as the nutrient levels. Borrowing from $E_{\max }$ theory [1], one choice is

$$
g(S, A)=f(S) \frac{A^{H}}{a^{H}+A^{H}}
$$

where $H>0$ is the Hill exponent, $a$ the half-saturation concentration and $f$ the specific growth rate. In this case, the net growth rate is

$$
f(S)-g(S, A)=f(S) \frac{a^{H}}{a^{H}+A^{H}}
$$

Cogan takes

$$
g(S, A)=k \frac{S+\alpha}{a+S} A
$$

where $\alpha=0$ for antibiotic that kills in proportion to growth rate (if $f(S)=m S / a+S$ ) and $\alpha>0$ for antibiotic which is partially effective in killing non-growing bacteria. The simplest term commonly used is independent of nutrient levels, namely

$$
g(S, A)=k A
$$

which is usually interpreted as kill rate. 
Commonly used functions appearing in the literature are described in the following table (table 1).

We make only qualitative assumptions regarding the pharmacodynamic function $g(S, A)$. It should vanish in the absence of drug and increase with antibiotic concentration:

$$
g(S, 0)=0, \quad \frac{\partial g}{\partial A} \geq 0
$$

Furthermore, adding nutrient should not decrease the net bacterial growth rate:

$$
S \rightarrow f(S)-g(S, A) \text { is nondecreasing for } 0 \leq S \leq S^{0}
$$

Finally, equation (1) includes a removal rate of antibiotic due to its association with bacteria, modeled by the term $-p(A) u$. $p$ might be taken to be Michaelis-Menten or more generally of Hill type, or simply $p(A)=c A$. As mentioned in the introduction, most pharmacodynamic models do not include such a term. In some cases, it may be reasonable to neglect this term entirely, taking $p=0$, if drug removal rate is relatively independent of $u$. This case is mathematically attractive since it decouples the pharmacokinetics from the rest of the model. We assume that $p$ vanishes with $A$ and is nondecreasing in $A$ :

$$
p(0)=0, \quad p^{\prime}(A) \geq 0
$$

The chemostat-based model requires considerable modification if we wish to view it as an in vivo model of the application of antibiotic to a tissue in an organism hosting an infectious bacteria. In this setting, the circulating blood may deliver the antibiotic to the infection site and the nutrient may be supplied by the infected tissue or by the blood circulation. For the in vivo interpretation, the assumption of an identical removal rate $D$ for all components is unreasonable. More generally, we consider

$$
\begin{aligned}
S^{\prime} & =d_{S} S^{0}-d_{S} S-\gamma^{-1} f(S) u \\
A^{\prime} & =d_{A} A_{0}(t)-d_{A} A-u p(A) \\
u^{\prime} & =\left[f(S)-d_{u}-g(S, A)\right] u \\
v^{\prime} & =g(S, A) u-d_{v} v
\end{aligned}
$$

where $F=d_{S} S^{0}$ is a flux of nutrient into the infected region, possibly supplied by surrounding tissues or the blood, and $d_{S}$ the removal rate of nutrient which might also include uptake by host cells. $d_{A} A_{0}(t)$ is now interpreted as flux of antibiotic into the infected region and $d_{A}$ its removal rate, perhaps due to metabolism in the liver and excretion by the kidney.

Table 1. Pharmacodynamic functions.

\begin{tabular}{lcl}
\hline Pharmacodynamic function & Reference & Description \\
\hline$g(A)=K a$ & {$[14]$} & Linear in $A$ \\
$g(A)=m \log (A)+I$ & {$[10]$} & Log form \\
$g(S, A)=k \frac{S}{a+S} A$ & {$[3]$} & Linear in $A$ \\
$g(A)=k \frac{A}{L^{H}+A^{H}}$ & {$[25,27]$} & $E_{\max }$ model \\
$g(A)=k \frac{A^{n}}{(L+A)^{n}}$ & $A$ & Binomial form \\
$g(A)=k \frac{A}{L_{0}+L_{1} A+L_{2} A^{2}+\ldots+L_{n} A^{n}}$ & {$[2]$} & Cooperativity form \\
\hline
\end{tabular}


As for equation (1), an additional antibiotic removal rate $-u p(A)$ due to association of drug with bacterial cells is included. In some cases, this term may be neglected if it is small in relation to removal by metabolism and excretion. The removal rate $d_{u}$ for viable bacteria $u$ may include effects of specific or non-specific immune response [1]. We have included an equation for $v$; the density of nonviable cells as the antibiotic may be bacteriostatic rather than bacteriocidal. However, as this equation is decoupled from the others, we ignore it hereafter.

Prior to antimicrobial treatment $A_{0}(t)=0$ and $A(t)=0$ so the model reduces to slight modification of the classical chemostat model of microbial growth on a single substrate [30].

$$
\begin{aligned}
& S^{\prime}=d_{S}\left(S^{0}-S\right)-\gamma^{-1} f(S) u \\
& u^{\prime}=\left[f(S)-d_{u}\right] u
\end{aligned}
$$

Assuming that $f\left(S^{0}\right)>d_{u}$ (otherwise, there is no need for treatment), the untreated infection steady state is given by

$$
S=\bar{S}, \quad u=\bar{u}=\gamma\left(S^{0}-\bar{S}\right), \quad f(\bar{S})=d_{u}
$$

This can be viewed as the pre-treatment state since this state is most likely approximated just prior to treatment.

If the effect of the antibiotic is to inhibit growth and uptake of nutrient then the following equations may be more natural for in vitro environments:

$$
\begin{aligned}
& S^{\prime}=D\left(S^{0}-S\right)-\gamma^{-1} f(S, A) u \\
& A^{\prime}=D\left(A_{0}(t)-A\right)-u p(A) \\
& u^{\prime}=(f(S, A)-D) u .
\end{aligned}
$$

where we always assume that the pharmacodynamic function $f(S, A) \geq 0$ is monotonically increasing in $S$ and monotonically decreasing in $A$. The function $f(S, A)$ could be chosen from among the following (table 2) where $\alpha(A)$ and $\beta(A)$ are in the units of concentration and time, respectively. $m$ denotes the maximum growth rate and $a$ is the Michaelis-Menton (or halfsaturation) constant:

The following equations are more appropriate for an in vivo environment:

$$
\begin{aligned}
& S^{\prime}=d_{S} S^{0}-d_{S} S-\gamma^{-1} f(S, A) u \\
& A^{\prime}=d_{A} A_{0}(t)-d_{A} A-u p(A) \\
& u^{\prime}=\left(f(S, A)-d_{u}\right) u .
\end{aligned}
$$

In our models (2) and (5), we assume the simplest possible pharmacokinetics, introducing only a single drug compartment $A$, representing the drug at the site of infection. As drug is

Table 2. Pharmacodynamic functions.

\begin{tabular}{lll}
\hline Pharmacodynamics & Reference & Description \\
\hline$f(S, A)=m \frac{S}{a+S} \exp (-x A)$ & {$[14,20]$} & Reduce maximum growth rate \\
$f(S, A)=m \frac{S}{(a+a(A))+S}$ & {$[4]$} & Reduce ability of bacteria to use substrate \\
$f(S, A)=(m-\beta(A)) \frac{S}{a+S}$ & {$[4]$} & Reduce maximum growth rate \\
\hline
\end{tabular}


usually administered orally or intravenously and then must pass through a number of organs and tissues before reaching the site of infection, more realistic models typically include additional compartments for drug levels in these tissues. Such extensions of our models could be included.

Let $T$ denote the length of the dosing period. If $g$ is a continuous $T$-periodic function then $[g]_{m}$ will denote the mean value of $g$ :

$$
[g]_{m}=\frac{1}{T} \int_{0}^{T} g(s) \mathrm{d} s
$$

Hereafter, all periodic functions are to be understood to be $T$-periodic unless otherwise noted.

It is natural to expect the existence of periodic responses to periodic antibiotic dosing; the constant dosing case is included as a special case. We expect two different types. Naturally, there is the "sterile state" or "infection-free state"

$$
E_{0}(t)=\left(S^{0}, A^{*}(t), 0\right)
$$

with no bacteria present and nutrient levels matching feed level. $A^{*}(t)$ is the unique periodic solution of

$$
A^{\prime}=d_{A} A_{0}(t)-d_{A} A
$$

$A^{*}(t)$ may be called the asymptotic pharmacokinetics since every solution of this simple differential equation is asymptotic to it as $t$ becomes large. It has the following properties

$$
\left[A^{*}\right]_{m}=\left[A_{0}\right]_{m}, \quad \min _{t} A_{0} \leq \min _{t} A^{*} \leq \max _{t} A^{*} \leq \max _{t} A_{0}
$$

The infection-free state may be viewed as the desired target state in the sense that successful treatment must drive the system state to it.

In addition, there may or may not be one or more "disease states" or "infection states" of the form

$$
E_{u}(t)=(\bar{S}(t), \bar{A}(t), \bar{u}(t))
$$

where $\bar{u}(t)>0$ and where all components are positive periodic functions. Such states correspond to treatment failure. Our results will focus on the existence, uniqueness and stability of these solutions.

The local stability of the sterile state can be determined by the Floquet exponents of the variational equation about $E_{0}(t)$. It turns out that two of these are negative; the third, the "invasion exponent", is given by

$$
\lambda=f\left(S^{0}\right)-d_{u}-\left[g\left(S^{0}, A^{*}(t)\right)\right]_{m}
$$

for system (2) and

$$
\lambda=\left[f\left(S^{0}, A^{*}(t)\right)\right]_{m}-d_{u}
$$

for system (5). In either case, $\lambda$ depends on the net, time-averaged bacterial growth rate in the asymptotic pharmacokinetic state where $S=S^{0}$ and $A=A^{*}(t)$, involving the growth dynamics, the pharmacodynamic function and the bacterial removal rate. 
The following theorem states our main result for both equations (2) and (5). It includes the autonomous case, $A_{0}=$ constant and the in vitro equations (1) and (4) as special cases.

Theorem 2.1. For systems (2) and (5), the sterile state $E_{0}(t)$ is locally asymptotically stable if $\lambda<0$ and unstable if $\lambda>0$. Moreover:

1. If $\lambda<0, E_{0}(t)$ is globally attracting in the following cases:

(i) for equation (5) when $p=0$ and for equation (4) if no $E_{u}(t)$ exists.

(ii) for equation (2) when $p=0$.

2. If $\lambda>0$, then bacteria persist. More precisely there exists $\varepsilon>0$, independent of initial data $(S(0), A(0), u(0))$ provided $u(0)>0$ and there exists $t_{0}>0$, depending on initial data, such that

$$
u(t)>\varepsilon, t>t_{0} .
$$

3. Furthermore, at least one infection state $E_{u}(t)$ exists. Assuming $\lambda>0$, the following also hold:

(iii) for equation (4), every solution with $u(0)>0$ converges to one of the periodic states $E_{u}(t)$; if $E_{u}(t)$ is unique, in particular when $p=0$, it attracts all solutions with $u(0)>0$.

(iv) If $A_{0}$ is constant and $p=0$ holds, then $E_{u}$ is unique and globally asymptotically stable for equation (2) and for equation (5).

In summary, Theorem 2.1 establishes that the invasion exponent $\lambda$, whose sign characterizes the local stability of the sterile state, can provide useful information concerning the global dynamics of the models in certain cases. Treatment failure is guaranteed when $\lambda>0$ in all cases since bacteria can grow when rare. Furthermore, there exists a least one (periodic) infection state $E_{u}(t)$; in general, it may be non-unique and we do not know its stability properties. An important exception is the case of constant dosing and $p=0$ where it is globally attracting.

Only in special cases have we shown that the reverse inequality $\lambda<0$ guarantees treatment success, in the sense of bacterial eradication, regardless of initial conditions. Both such cases require that the removal rate of antibiotic be independent of bacterial density $(p=0)$.

We note that the system (4) is much more mathematically tractable due to a conservation principle common to chemostat models [30]; the corresponding reduced system has special monotonicity properties.

The left side of figure 1 depicts periodic dosing of an antibiotic, given every four hours, with a mean value of 0.2 . The right side describes the asymptotic pharmacokinetics $A^{*}(t)$ after initial transients die out. Parameters were chosen as in Ref. [27].

Our simulations use only equation (2) with

$$
f(S)=\frac{m S}{a+S}, \quad p(A)=\frac{\nu A}{L_{1}+A} \quad \text { and } \quad g(S, A)=k \frac{S}{a+S} \frac{A}{L+A} .
$$

The left side of figure 2 shows successful treatment and the right figure depicts unsuccessful treatment. All the parameters and functions are the same in both the figures except the maximum killing rate and it is chosen in such a way that $\lambda<0$ in the left figure and $\lambda>0$ in the right. Output has been scaled by $S / a, u /(a \gamma), A / L$. Time $t$ is scaled by $1 / d_{S}, S^{0}$ is scaled by $1 /(a), A_{0}$ is scaled by $1 / L$ and $d_{A}, d_{u}, m$ and $k$ are all scaled by $1 / d_{S}$. Parameter values are 

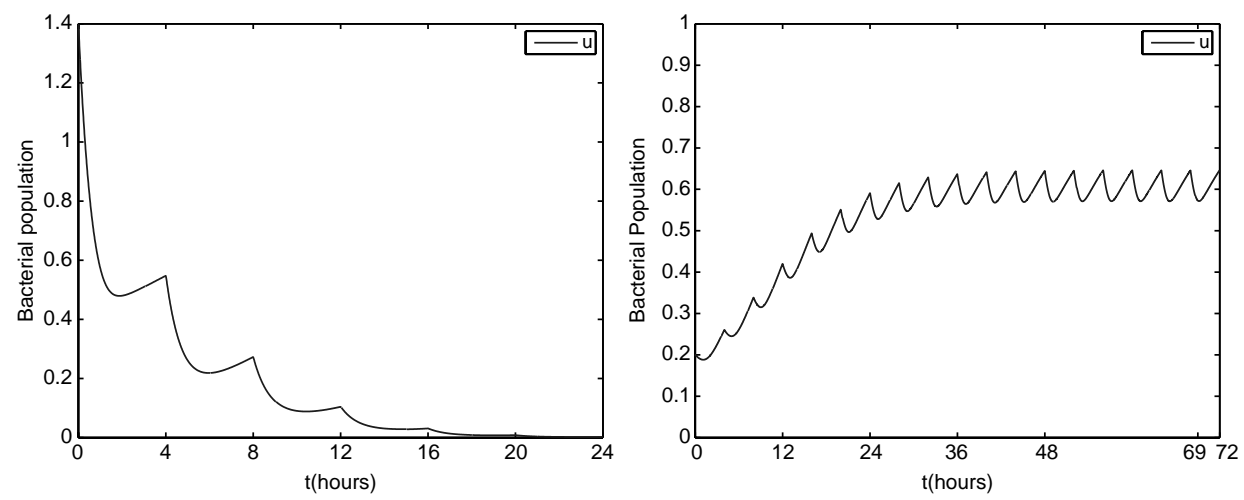

Figure 2. The left figure shows treatment success when $\lambda=-0.2804<0$. The right depicts treatment failure when $\lambda=0.1555>0$. Same dosing as figure 1 .

chosen as in Refs. [3,17,28]. In particular: yield coefficient $\gamma=0.8$; maximum specific growth rates $m=0.417, \nu=0.28$; dilution rate $d_{S}=0.23$; half saturation constants $a=0.1$, $L=0.1$ and $L_{1}=0.1$; maximum disinfection rate $k=0.96$ (left) and $k=0.13$ (right); concentration of the substrate in the feed $S^{0}=0.2$; and Hill exponent $H=1$ (figure 3).

Figure 4 illustrates that $\lambda<0$ only guarantees successful treatment of small bacterial populations except in certain special cases. The bacterial population versus time is plotted for a small and for a large initial inoculum of bacteria $(u(0)=0.25$ and $u(0)=0.45)$ at the start of a treatment; all other parameters and initial data are the same. The solution corresponding to the small bacterial inoculum shows successful treatment while the solution corresponding to a large initial bacterial level exhibits treatment failure. Both $E_{0}(t)$ and $E_{u}(t)$ are simultaneously locally stable. All parameters are the same as used in the previous figure except $k=0.96$ and $S^{0}$ is increased to $S^{0}=0.5$. The phenomena of bistability may have important implications for antibiotic treatment and appears not to have been observed in

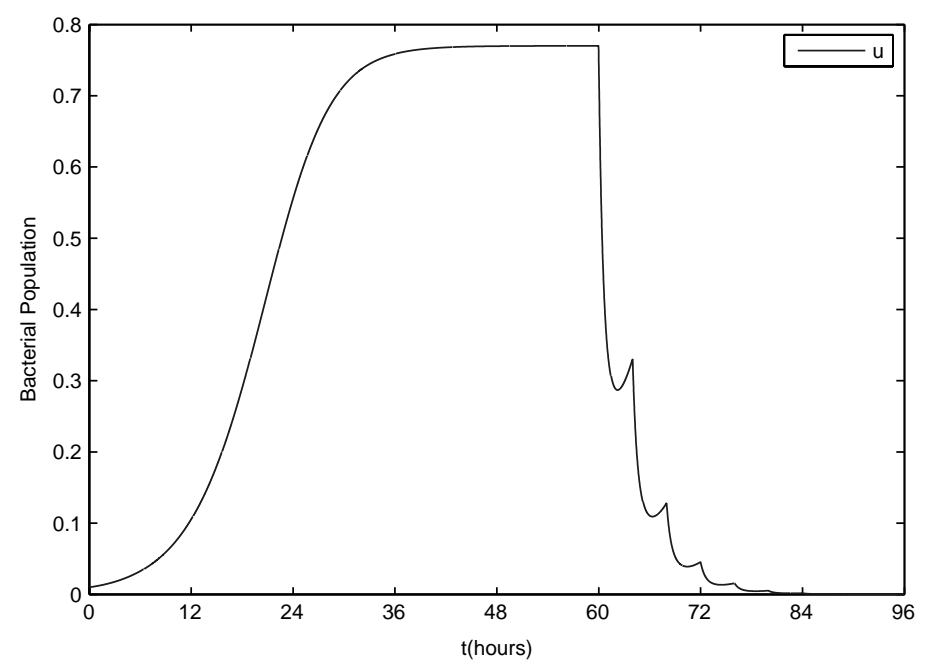

Figure 3. An illustration of pre-treatment infection for $0<t<60 \mathrm{~h}$ followed by treatment starting at $t=60 \mathrm{~h}$ resulting in bacterial eradication. Same dosing as figure 1. 


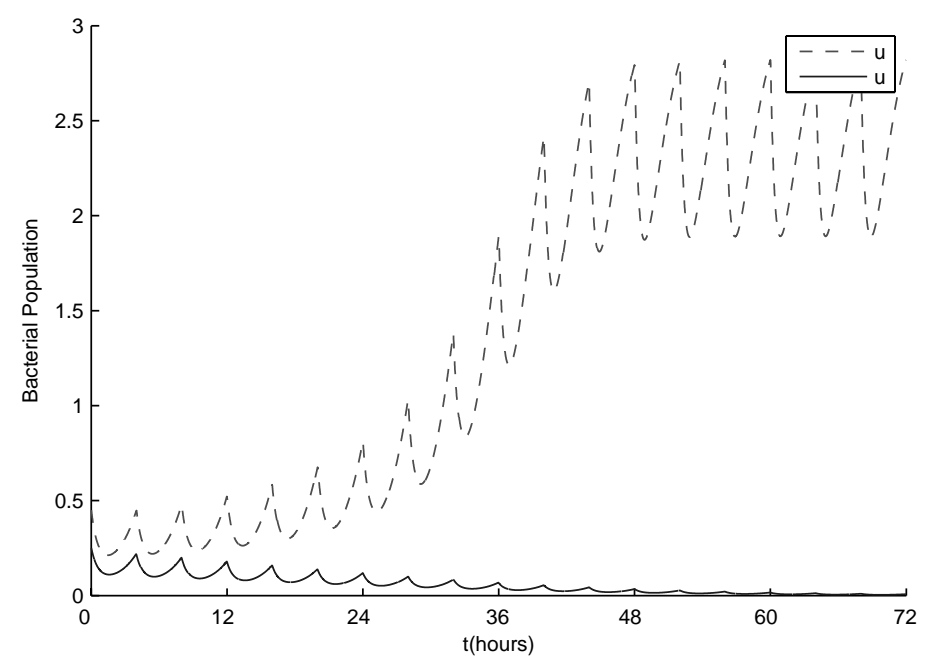

Figure 4. Initial-condition-dependent outcomes are possible when $\lambda<0$ : successful and unsuccessful treatment result from the same system but different initial data. Same dosing as figure 1 .

earlier modeling work. It suggests that early treatment, before bacterial populations become large, is best.

Figures 5 and 6 consider the constant dosing case for equation (2). Figure 5 (left) plots the scaled value of $u$ at steady state $E_{u}$ versus the constant dosing concentration of antibiotic $A_{0}$. Parameter values are the same used in previous figures except for $S^{0}$ and $k$, which are $S=0.5$ and $k=0.45$. The bifurcating branch $E_{u}$ is stable from $\left(u, A_{0}\right)=(3.8,0)$ to LP and is unstable from LP to $(0,0.44)$. This figure shows that although the concentration of antibiotic exceeds the MIC, treatment is unsuccessful. If we increase the initial concentration of antibiotic more than 0.67 then the bacteria population will be cleared from the host. The number of infection steady states as a function of input parameters $S^{0}$ and $A_{0}$ is shown in the right side of figure 5. The MIC curve $\lambda=0$ is also plotted, showing that there is only one
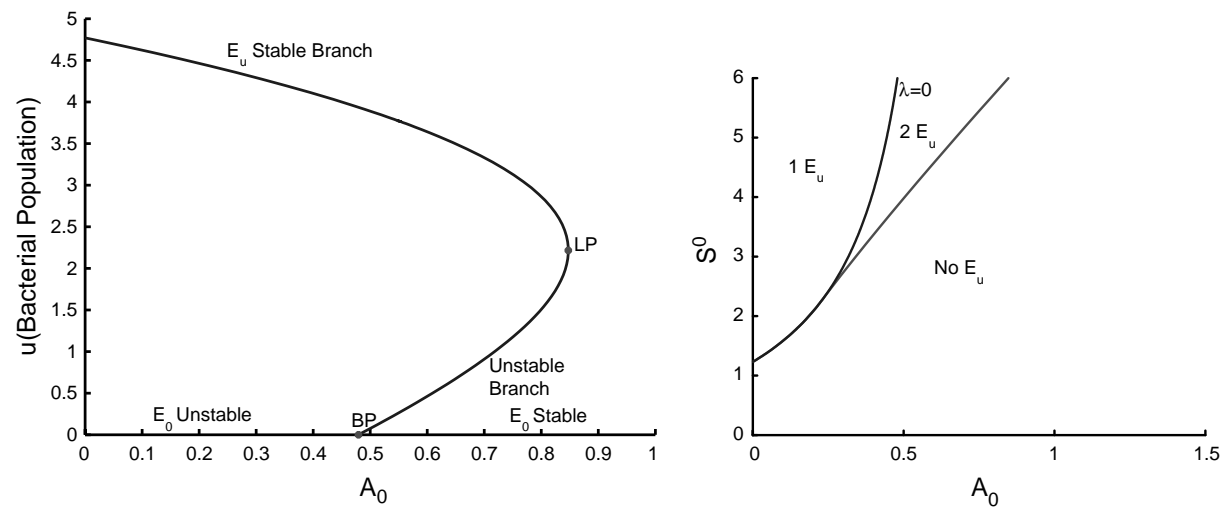

Figure 5. Bifurcation diagram for constant dosing on left shows the existence of a large-amplitude, stable branch of $E_{u}$ while $E_{0}$ is stable; $A_{0}$ is the bifurcation parameter. The right figure shows the MIC $(\lambda=0)$ curve and regions corresponding to different numbers of disease steady states in the $\left(A_{0}, S^{0}\right)$-plane for constant dosing. 


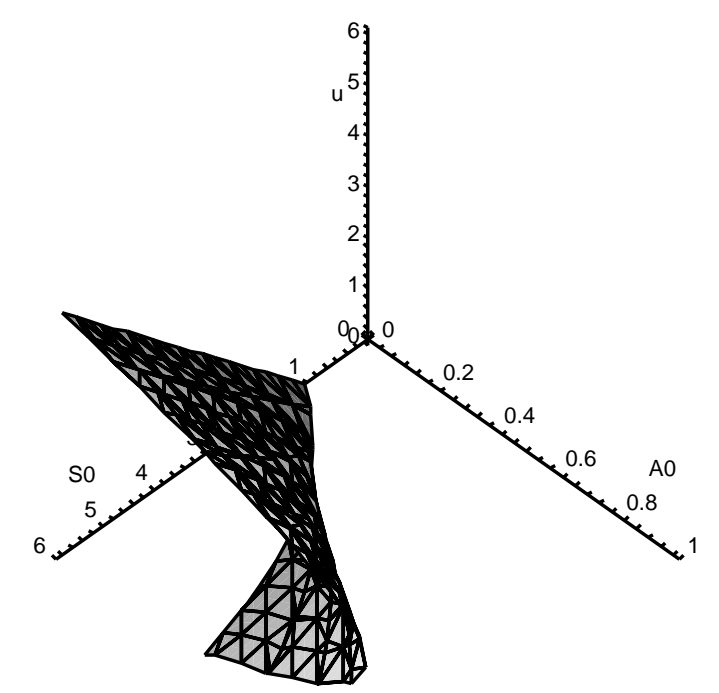

Figure 6. The dependence of the infection steady state $\left(E_{u}\right)$ value of $u$ on parameters $\left(S^{0}, A_{0}\right)$ for constant dosing, illuminating figure 5 . The surface intersects the $u=0$ plane along the $\lambda=0$ curve.

disease state when concentration of antibiotic is less than the MIC value, and zero or two disease states when concentration is more than MIC.

Figure 6 shows the surface $u=u\left(S^{0}, A_{0}\right)$, giving the bacterial density at the infection state $E_{u}$. A fold in this surface illuminates the information contained in the right side of figure 5.

Theorem 2.1 does not provide an adequate set of sufficient conditions for treatment success in the general case $p \neq 0$. Our next result applies much more generally, providing a threshold condition for treatment success in terms of parameters and functions appearing in the model. It is a special case of a more general result proved in the appendix.

Theorem 2.2. Let

$$
B_{0}=\frac{\gamma d_{S} S^{0}}{\min \left\{d_{S}, d_{u}\right\}}
$$

and suppose that

$$
p(A) \leq c A, \quad 0 \leq A \leq \max _{t} A_{0}(t)
$$

If, in addition, $A \rightarrow g\left(S^{0}, A\right)$ is concave on $0 \leq A \leq \max _{t} A_{0}(t)\left(\right.$ e.g. $\left.\left(\partial^{2} g / \partial A^{2}\right) \leq 0\right)$ and

$$
f\left(S^{0}\right)-d_{u}-g\left(S^{0}, \frac{d_{A}}{d_{A}+B_{0} c}\left[A_{0}\right]_{m}\right)<0
$$

then $u(t) \rightarrow 0$ as $t \rightarrow \infty$ for every solution of equation (2).

The concavity assumption on $g$ is not necessary but it simplifies the statement of the result. It is satisfied for many of the functions commonly used including the $E_{\max }$ model with exponent one. Analogous results can be proved for equation (5). Assumption (6) is a mild one as $c$ can be taken to be $\sup \left\{p(A) / A: 0<A \leq \max A_{0}(t)\right\}$ so long as $p$ is differentiable at $A=0$. Note that the expression on the left in equation (7), which exceeds $\lambda$, depends only on the supplied antibiotic level. 


\section{Treatment of susceptible and resistant bacteria}

Bacteria may acquire resistance to an antibiotic via spontaneous mutation or the acquisition of new genetic material by transduction, transformation or conjugation. Transduction is the process by which bacterial DNA is moved from one bacterium to another by a virus. Transformation is a process where pieces of DNA in the exterior environment are taken up by the bacteria. Conjugation is the transfer of genetic material between bacteria through cell-tocell contact and it is thought to play a significant role in the proliferation of antibiotic resistant pathogens [29,34]. We consider a bacterial population consisting of two types of cells: a plasmid-free cell which is susceptible to an antibiotic and a plasmid-bearing cell which is partially or totally resistant to the antibiotic due to a resistance gene encoded by the plasmid. Hereafter, we refer to the cell types as resistant and susceptible. We assume that the resistant, plasmid-bearing cell may transfer a copy of the plasmid to a susceptible, plasmidfree cell via conjugation. Furthermore, a plasmid-bearing cell may mis-segregate plasmid at cell division with probability $q$ resulting in one daughter cell receiving no plasmid and hence becoming susceptible. Our model takes the form:

$$
\begin{aligned}
S^{\prime} & =d_{S}\left(S^{0}-S\right)-\gamma^{-1}\left[f(S) u+f_{+}(S) u_{+}\right] \\
A^{\prime} & =d_{A}\left(A_{0}(t)-A\right)-p(A)\left[u+u_{+}\right] \\
u^{\prime} & =\left[f(S)-d_{u}-g(S, A)\right] u+q f_{+}(S) u_{+}-\mu u u_{+} \\
u_{+} & =\left[f_{+}(S)(1-q)-d_{u}-g_{+}(S, A)\right] u_{+}+\mu u u_{+}
\end{aligned}
$$

For simplicity, we assume both cells types have the same growth yield and removal rate and remove antibiotic similarly. We expect that resistant cells grow no faster than susceptible ones and of course, are at least partially resistant to antibiotic:

$$
f_{+}(S) \leq f(S) \quad \text { and } \quad g_{+}(S, A)<g(S, A)
$$

Such a cost of plasmid carriage is well documented in the literature, see Lenski [21] although it may decline over many generations due to the effects of natural selection as observed by Dahlberg and Chao [8].

Horizontal transmission of the plasmid between the two sub-populations is assumed to occur at a rate proportional to the product of their densities: $\mu и u_{+}$.

Our model equation (8) is based on the plasmid model of Stephanopoulus and Lapidus [33]. See also Hsu et al. [11-14] for a similar modeling of segregative loss in their model of competition between plasmid-bearing and plasmid-free organisms in selective media and Refs. [17,18] for plasmid transfer in biofilms.

Removing the antibiotic from the model results in the "pre-treatment model"

$$
\begin{aligned}
S^{\prime} & =d_{S}\left(S^{0}-S\right)-\gamma^{-1}\left[f(S) u+f_{+}(S) u_{+}\right] \\
u^{\prime} & \left.=\left[f(S)-d_{u}\right)\right] u+q f_{+}(S) u_{+}-\mu u u_{+} \\
u_{+} & =\left[f_{+}(S)(1-q)-d_{u}\right] u_{+}+\mu u u_{+} .
\end{aligned}
$$

which is studied in detail in Ref. [18] in the case $d_{S}=d_{u}$. If $u_{+}=0$, it reduces to equation (3) and if $f\left(S^{0}\right)>d_{u}$ it has the pretreatment steady state $(\bar{S}, \bar{u}, 0)$ with no resistant bacteria which is locally stable for equation (9) if $f_{+}(\bar{S})(1-q)-d_{u}+\mu \bar{u}<0$ and unstable if the reverse inequality holds. If $f_{+}(\bar{S})(1-q)-d_{u}+\mu \bar{u}>0$, then equation (9) has another pretreatment 
state $(\tilde{S}, \tilde{u}, \tilde{u}+)$ consisting of both susceptible and resistant bacteria. Appropriate initial conditions for the post-treatment system (8) are expected to be near the stable equilibrium of the pre-treatment system (9).

If the antibiotic inhibits the growth and uptake of the nutrient then the following equations may be more appropriate

$$
\begin{aligned}
S^{\prime} & =d_{S}\left(S^{0}-S\right)-\gamma^{-1}\left[f(S, A) u+f_{+}(S, A) u_{+}\right] \\
A^{\prime} & =d_{A}\left(A_{0}(t)-A\right)-p(A)\left[u+u_{+}\right] \\
u^{\prime} & =\left[f(S, A)-d_{u}\right] u+q f_{+}(S, A) u_{+}-\mu u u_{+} \\
u_{+} & =\left[f_{+}(S, A)(1-q)-d_{u}\right] u_{+}+\mu u u_{+} .
\end{aligned}
$$

If the resistance is due to spontaneous mutation, then the above model (8) can be written as

$$
\begin{aligned}
& S^{\prime}=d_{S}\left(S^{0}-S\right)-\gamma^{-1}\left[f(S) u+f_{+}(S) u_{+}\right] \\
& A^{\prime}=d_{A}\left(A_{0}(t)-A\right)-p(A)\left[u+u_{+}\right] \\
& u^{\prime}=\left[f(S)-d_{u}-g(S, A)\right] u-q u \\
& u_{+}=\left[f_{+}(S)-d_{u}-g_{+}(S, A)\right] u_{+}+q u .
\end{aligned}
$$

where $q$ is the mutation rate from susceptible to resistant.

The periodic solutions of equations (8) and (10) include the "sterile state"

$$
E_{0}(t)=\left(S^{0}, A^{*}(t), 0,0\right)
$$

"infection states" with only susceptible cells of the form

$$
E_{u}(t)=(\bar{S}(t), \bar{A}(t), \bar{u}(t), 0)
$$

and infection states with susceptible and resistant organisms of the form

$$
E_{u^{*}}(t)=\left(S^{*}(t), A^{*}(t), u^{*}(t), u_{+}^{*}(t)\right)
$$

where all components are positive periodic functions. Such infection states need not be unique. Note that if $u_{+}(0)=0$ then $u_{+}(t)=0$ for all $t$ and this case is discussed in the previous section. Therefore we assume that $u(0)>0$ and $u_{+}(0)>0$.

As in the previous section, the local stability of the sterile state can be determined by Floquet exponents of the variational equation associated with the periodic solution $E_{0}(t)$. Two of these, $-d_{S},-d_{A}$ are negative; the third and the fourth are the invasion exponents for susceptible and resistant organisms:

$$
\lambda=f\left(S^{0}\right)-d_{u}-\left[g\left(S^{0}, A^{*}(t)\right)\right]_{m}, \quad \lambda_{+}=f_{+}\left(S^{0}\right)(1-q)-d_{u}-\left[g_{+}\left(S^{0}, A^{*}(t)\right)\right]_{m}
$$

for system (8) and

$$
\lambda=\left[f\left(S^{0}, A^{*}(t)\right)\right]_{m}-d_{u}, \quad \lambda_{+}=\left[f_{+}\left(S^{0}, A^{*}(t)\right)\right]_{m}(1-q)-d_{u}
$$

for system (10).

The counterpart to Theorem 2.1 for treatment of susceptible and resistant populations is the following result. 
Theorem 3.1. For systems (8) and (10), the sterile state $E_{0}(t)$ is locally asymptotically stable if both $\lambda<0$ and $\lambda_{+}<0$ and unstable if either $\lambda>0$ or $\lambda_{+}>0$. Moreover:

1. If $\lambda<0, A_{0}$ is constant and $p=0$, then $E_{0}$ is globally attracting for equation (8) when $f_{+}\left(S^{0}\right)-d_{u}-g_{+}\left(S^{0}, A_{0}\right)<0$ and for equation (10) when $f_{+}\left(S^{0}, A_{0}\right)-d_{u}<0$.

2. If $\lambda>0$ or $\lambda_{+}>0$, then treatment fails. In particular, there exists $\varepsilon>0$, independent of initial data and $t_{0}>0$ depending on initial data, such that

$$
u(t)+u_{+}(t)>\varepsilon, \quad t>t_{0}
$$

3. At least one infection state $E_{u}(t)$ or $E_{u^{*}}(t)$ exists. If resistant population survive then so does susceptible, in the sense that if equation (11) holds with only $u_{+}$, then a similar statement holds for $u$. Assuming $\lambda<0, \lambda_{+}>0$ and $p=0$, then both $u$ and $u_{+}$are uniformly persistent for equations (8) and (10) and at least one infection state $E_{u^{*}}$ exists.

4. If $A_{0}$ is constant,$\lambda>0$ and $p=0$ then $E_{u}=(\bar{S}, \bar{A}, \bar{u}, 0)$ exists, is unique and is locally asymptotically stable for equation (8) if $\bar{\lambda}_{+}=f_{+}(\bar{S})(1-q)-d_{u}-g_{+}(\bar{S}, \bar{A})+\mu \bar{u}<0$ and for equation (10) if $\bar{\lambda}_{+}=f_{+}(\bar{S}, \bar{A})(1-q)-d_{u}+\mu \bar{u}<0 . E_{u}$ is unstable if $\bar{\lambda}_{+}>0$; in this case $u$ and $u_{+}$uniformly persist and $E_{u^{*}}$ exists for equations (8) and (10).

Theorem 3.1 describes the extent to which we have been able to link the local stability properties of the sterile state, as determined by the sign of the invasion exponents $\lambda$ and $\lambda_{+}$, to the global dynamics of the models. In the case of non-constant, periodic dosing, the diseasefree state is only locally stable when both $\lambda<0$ and $\lambda_{+}<0$. This means treatment success is only guaranteed for indeterminately small infections. On the other hand, treatment failure is guaranteed when either $\lambda>0$ or $\lambda_{+}>0$, in which case there exists at least one periodic disease state $E_{u}(t)$ or $E_{u^{*}}(t)$. Special attention is focused on the case $p=0$ with $\lambda<0$ and $\lambda_{+}>0$ where the resistant, but not the susceptible, strain can invade the sterile state. Of course, treatment failure occurs, both resistant and susceptible organisms persist and there is a periodic disease state $E_{u^{*}}$ with both resistant and susceptible populations.

More information is available for the constant dosing case. In particular, global stability of the disease-free state holds under certain conditions when $\lambda<0$ and $p=0$. If $\lambda>0$ and $p=0$, then $E_{u}$ exists and is unique, and its stability is determined by whether or not $u_{+}$can successfully invade it, as determined by the sign of $\bar{\lambda}_{+}$. If the latter is positive then both resistant and susceptible bacteria persist and there is a corresponding steady state with both present.

Our simulation in this section involves only equation (8) with $f(S), p(A), g(S, A)$ as defined in the previous section and

$$
f_{+}(S)=\frac{m_{+} S}{a+S}, \quad g_{+}(S, A)=k_{+} \frac{S}{a+S} \frac{A}{L+A} .
$$

Figure 7 depicts a successful treatment when both $\lambda$ and $\lambda_{+}$are negative. The output has been scaled by $S / a, A / L, u /(a \gamma)$ and $u_{+} /(a \gamma)$. Time $t$ is scaled by $1 / d_{S}, \nu$ is measured in units of $(a \gamma) /\left(d_{S} L\right), \mu$ is measured in units of $a \gamma, d_{A}, d_{u}, m, m_{+}, k$ and $k_{+}$are all scaled by $1 / d_{S}, S^{0}$ is scaled by $1 /(a \gamma)$ and $A_{0}$ is scaled by $1 / L$. Parameter values are: yield coefficient $\gamma=0.8$, maximum specific growth rates $m=0.417, m_{+}=0.416$ and $\nu=0.345$, dilution rate $d_{S}=0.23$, half saturation constants $a=0.1$ and $L=0.1$, Hill exponent $H=1$, maximum disinfection rate $k=0.96$ and $k_{+}=0.87$, concentration of the substrate in the feed $S^{0}=0.2$, segregation loss $q=0.01$ and conjugation $\mu=0.0000001$. 

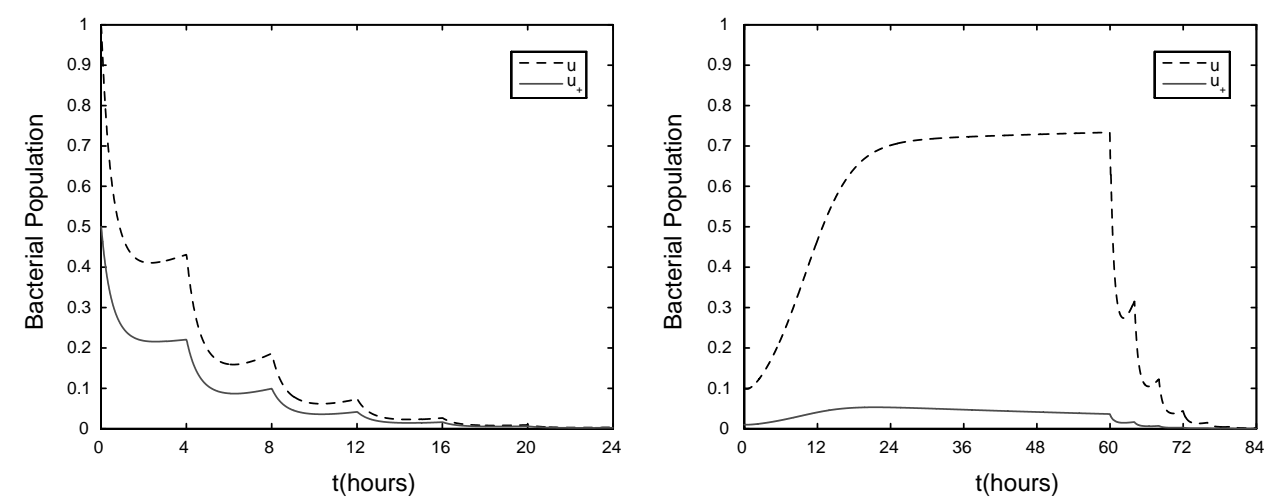

Figure 7. The left figure shows treatment success when $\lambda=-0.2804$ and $\lambda_{+}=-0.2511$. The right figure is an illustration of pre-treatment infection for $0<t<60 \mathrm{~h}$ followed by treatment starting at $t=60 \mathrm{~h}$ resulting in bacterial eradication. The value of $\lambda$ and $\lambda_{+}$are as in left figure during the treatment. Same dosing as figure 1 .

Figure 8 shows that the treatment could fail either by $\lambda_{+}>0$ and $\lambda<0$ or $\lambda>0$ and $\lambda_{+}>0$. Initially the culture contains the mixture with a minority of resistant bacteria and a majority of sensitive bacteria. In the first case (left figure), the susceptible population declines for first $24 \mathrm{~h}$ and then increases. In the second case, the susceptible population grows for first $24 \mathrm{~h}$ and then starts decreasing before reaching a periodic solution and the resistant population grows. In both cases, the resistant population becomes dominant and overcomes the susceptible population because the killing rate of resistant population is much smaller than for the susceptible one. All the parameters are the same as used in the previous figure, except $k$ and $k_{+}$are now 0.96 and 0.25 for figure (a) and 0.29 and 0.125 for figure (b).

Figure 9 shows that $\lambda<0$ and $\lambda_{+}<0$ only guarantees successful treatment of small bacterial populations except in certain special cases. Both resistant and susceptible populations versus time are plotted for a small and for a large initial inoculum of bacteria $\left(u(0)=0.5, u_{+}(0)=0.01\right.$ and $\left.u(0)=0.7, u_{+}(0)=0.01\right)$. All other parameters and initial data are the same as in previous figures. Solutions corresponding to small initial data show successful treatment while solutions corresponding to a large initial bacterial populations exhibit treatment failure. In the failure case, the susceptible population grows and takes over
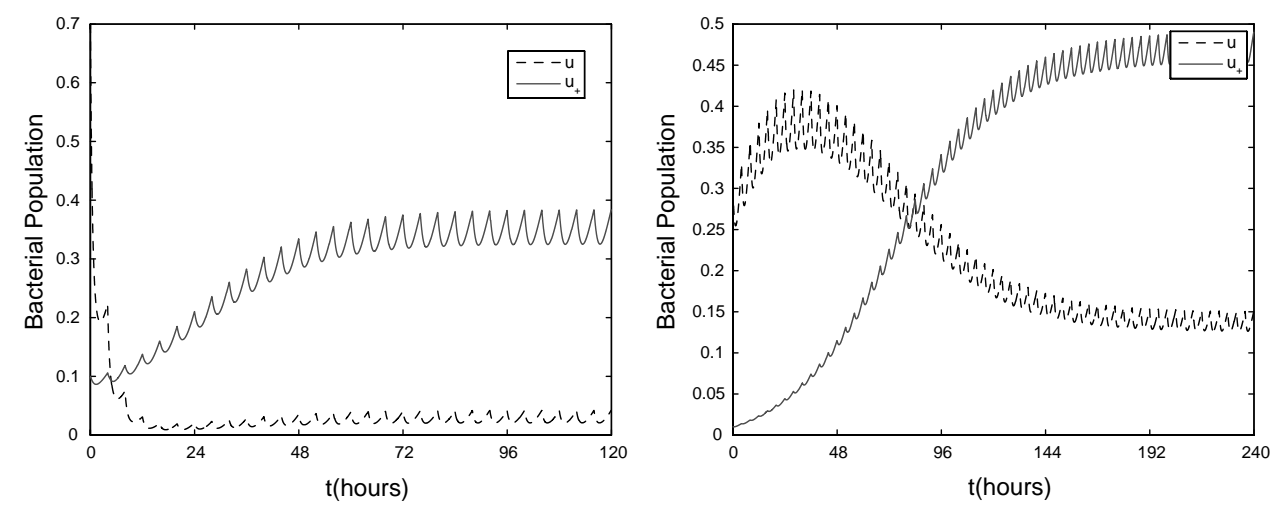

Figure 8. Simulation of the effect of unsuccessful treatment on the bacterial population. (Left) $\lambda=-0.3654$ is negative and $\lambda_{+}=0.0558$ is positive. In this case both populations persists and the treatment is ineffective. (Right) Both $\lambda=0.0598, \lambda_{+}=0.1316$ are positive and the treatment is ineffective. Same dosing as figure 1 . 

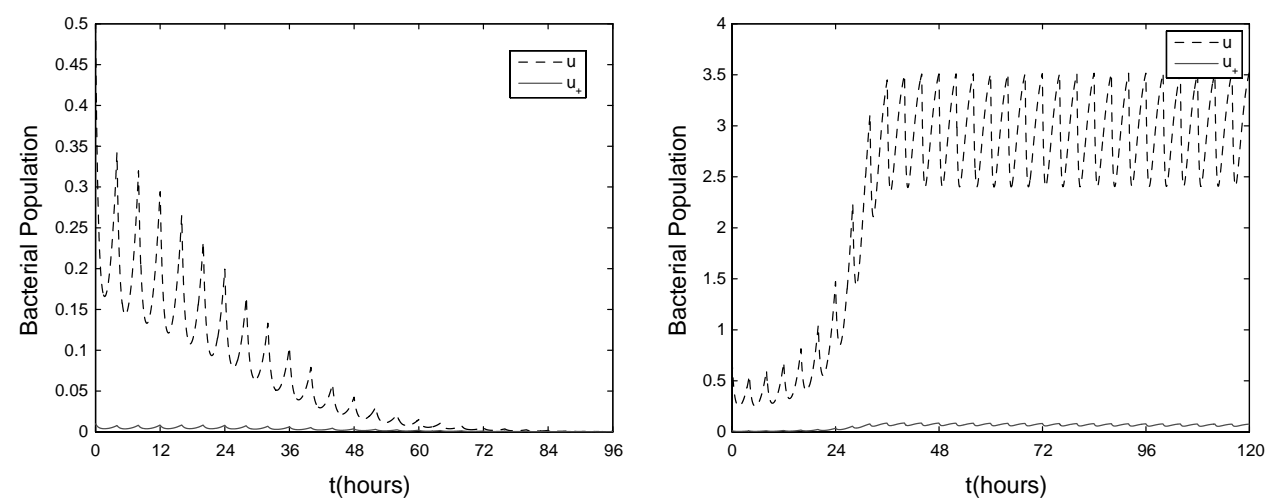

Figure 9. Simulation of the effect of treatment on the bacterial population. In both the figures $\lambda$ and $\lambda_{+}$are negative. The right figure shows a successful treatment and the left figure shows an unsuccessful treatment. Same dosing as figure 1.

the resistant population. All parameters are the same as used in left figure 7 except $S^{0}$ is increased to $S^{0}=0.5$.

The following analog of Theorem 2.2 gives simple sufficient conditions guaranteeing treatment success. It is a special case of a more general result proved in an appendix.

Theorem 3.2. Let $B_{0}$ and $c$ be as in Theorem 2.2. Suppose that $f_{+} \leq f, g_{+} \leq g$ and in addition, $A \rightarrow g_{+}\left(S^{0}, A\right)$ is concave on $\left[0, \max _{t} A_{0}(t)\right]$ (e.g. $\left.\left(\partial^{2} g_{+} / \partial A^{2}\right) \leq 0\right)$. Finally, assume that

$$
\lambda_{*}=f\left(S^{0}\right)-d_{u}-g_{+}\left(S^{0}, \frac{d_{A}}{d_{A}+B_{0} c}\left[A_{0}\right]_{m}\right)<0
$$

Then $u(t)+u_{+}(t) \rightarrow 0$ as $t \rightarrow \infty$ for every solution of equation (8).

Observe that $\lambda_{*}$ appearing in equation (12) depends on the (larger) specific growth rate of susceptible bacteria and the (smaller) pharmacodynamic function of resistant bacteria.

\section{References}

[1] Austin, D.J., White, N.J. and Anderson, R.M., 1998, The dynamics of drug action on the within-host population growth of infectious agents: melding pharmacokinetics with pathogen population dynamics, Journal of Theoretical Biology, 194(3), 313-339.

[2] Chappell, M.J., 1995, Structure identifiability and indistinguishability of certain two-compartment models incorporating nonlinear efflux from the peripheral compartment, Mathematical Biosciences, 125, 61-81.

[3] Cogan, N.G., 2006, Persisters, tolerance and dosing, Journal of Theoretical Biology, 238(3), 694-703.

[4] Corpet, D.E., Lumeau, S. and Corpet, F., 1989, Minimum antibiotics levels for selecting a resistance plasmid in a gnotobiotic animal model, Antimicrobial Agents and Chemotherapy, 33(4), 535-540.

[5] Corvaisier, S., Maire, P., Bouvier D’Yvoire, M., Barbaut, X., Bleyzac, N. and Jelliffe, R., 1998, Comparisons between antimicrobial pharmacodynamic indices and bacterial killing as described by using the Zhi model, Antimicrobial Agents and Chemotherapy, 42(7), 1731-1737.

[6] Cozens, R.M., Tuomanen, E., Tosch, W., Zak, O., Suter, J. and Tomasz, A., 1986, Evaluation of the bactericidal activity of beta-lactam antibiotics on slowly growing bacteria cultured in the chemostat, Antimicrobial Agents and Chemotherapy, 29(5). 
[7] Craig, W.A., 1998, Pharmacokinetics/pharmacodynamic parameters: rationale for antibacterial dosing of mice and men, Clinical Infectious Diseases, 26, 1-12.

[8] Dahlberg, C. and Chao, L., 2003, Amelioration of the cost of conjugative plasmid carriage in Eschericha coli K12, Genetics, 165, 1641-1649.

[9] Hale, J.K., 1980, Ordinary Differential Equations, 2nd ed. R.E. Krieger Publishing Company, INC.).

[10] Nicholas, H., Holford, G. and Sheiner, L.B., 1982, Kinetics of pharmacologic response, Pharmacology \& Therapeuticss, 16, 143-166.

[11] Butler, G.J., Hsu, S.B. and Waltman, P., 1985, A mathematical model of the chemostat with periodic washout Rate, SIAM Journal on Applied Mathematics, 45(3), 435-449.

[12] Hsu, S.B., Waltman, P. and Wolkowicz, G., 1994, Global analysis of a model of plasmid-bearing, plasmid-free competition in the chemostat, Journal of Mathematical Biology, (32), 731-742.

[13] Hsu, S.B. and Waltman, P., 1997, Competition between plasmid-bearing and plasmid-free organisms in selective media, Chemical Engineering Science, 52, 23-35.

[14] Hsu, S.B. and Waltman, P., 2004, A survey of mathematical models of competition with an inhibitor, Mathematical Biosciences, 187, 53-91.

[15] Lipsitch, M. and Levin, B.R., 1997, The population dynamics of antimicrobial chemotherapy, Antimicrobial Agents and Chemotherapy, 41(2), 363-373.

[16] Hofbaer, J. and So, J.W.-H., 1989, American Mathematical Society Proceedings of the American Mathematical Society, 107(4).

[17] Imran, M., Jones, D. and Smith, H.L., 2005, Biofilms and the plasmid maintenance question, Mathematical Biosciences, 193, 183-204.

[18] Imran, M. and Smith, H.L., 2006, A mathematical model of gene transfer in a biofilm. Mathematics for Ecology and Environmental Sciences Spinger book, vol. 1 (accepted).

[19] Imran, M., 2006, Mathematical models in biofilms and antibiotic treatment, Ph.D. thesis, Arizona State University, Tempe, AZ.

[20] Lenski, R. and Hattingh, S., 1986, Coexistence of two competitors on one resource and one inhibitor, Journal Theoretical Biology, 122, 83-92.

[21] Lenski, R., 1998, Bacterial evolution and the cost of antibiotic resistance, International Microbiology, 1, $265-270$.

[22] Lewis, K., 2001, Riddle of biofilm resistence, Antimicrobial Agents and Chemotherapy, 45(4), 999-1007.

[23] Kuznetsov, Y.A., 1995, Elements of Applied Bifurcation Theory (New York: Springer-Verlag).

[24] Macheras, P. and Iliadis, A., 2006, Modeling in Biopharmaceutics, Pharmacokinetics and Pharmacodynamics Homogeneous and Heterogeneous Approaches (New York: Springer).

[25] Nikolaou, M. and Tam, V.H., 2006, A new modeling approach to the effect of antimicrobial agents on heterogeneous microbial population, Journal of Mathematical Biology, 52, 154-182.

[26] Odenholt, I., Lowdin, E. and Cars, O., 1997, Studies of the killing kinetics of benzylpenicillin, cefuroxime, azithromycin and sparfloxacin on bacteria in the postantibiotic phase, Antimicrobial Agents and Chemotherapy, 41(11), 2522-2526.

[27] Regoes, R.R., Wiuff, C., Zappala, R.M., Garner, K.N., Baquero, F. and Levin, B.R., 2004, Pharmacodynamic functions: a multiparameter approach to the design of antibiotic treatment regimens, Antimicrobial Agents and Chemotherapy, 48(10), 3670-3676.

[28] Roberts, M.E. and Stewart, P.S., 2004, Modeling antibiotic tolerance in biofilms by accounting for nutrient limitation, Antimicrobial Agents and Chemotherapy, 48(1), 48-52.

[29] Schwalbe, R.S., Hoge, C.W., Morris, J.G., O'Hanlon, P.N., Crawford, R.A. and Gilligan, P.H., 1990, In vivo selection for transmissible drug resistance in salmonella typhi during antimicrobial therapy, Antimicrobial Agents and Chemotherapy, 34(1), 161-163.

[30] Smith, H.L. and Waltman, P., 1995, The Theory of the Chemostat (New York: Cambridge University Press).

[31] Smith, H.L., 1986, Cooperative systems of differential equations with concave nonlinearties, Nonlinear Analysis: Theory, Methods \& Applications, 10, 1037-1052.

[32] Stemmons, E. and Smith, H.L., 2000, Competition in a chemostat with wall attachment, SIAM Journal on Applied Mathematics, 61, 567-595.

[33] Stephanopoulus, G. and Lapidus, G., 1988, Chemostat dynamics of plasmid-bearing plasmid-free mixed recombinant cultures, Chemical Engineering Science, 43, 49-57.

[34] Summers, D., 1996, The Biology of Plasmids (London: Blackwell Science).

[35] Thieme, H.R., 1993, Persistence under relaxed point-dissipativity (with application to an epidemic model), SIAM Journal on Mathematical Analysis, 24, 407-435.

[36] Tuomanen, E., 1986, Phenotypic tolerance: the search for beta-lactam antibiotics that kill nongrowing bacteria, Reviews of Infectious Disease, 8(3), 279-291.

[37] Tuomanen, E., Cozens, R., Tosch, W., Zak, O. and Tomasz, A., 1986, The rate of killing of Escherichia coli by beta-lactam antibiotics is strictly proportional to the rate of bacterial growth, Journal of General Microbiology, 132(5), 1297-1304. 
[38] Wiuff, C., Zappala, R.M., Regoes, R.R., Garner, K., Baquero, F. and Levin, B.R., 2005, Phenotypic tolerance: antibiotic enrichment of noninherited resistance in bacterial populations, Antimicrobial Agents and Chemotherapy, 49(4), 1483-1494.

[39] Zhao, X.-Q., 2003, Dynamical systems in population biology. CMS Books in Mathematics (New York: Springer).

\section{A. Appendices}

Proofs of our results are provided in these appendices. The proof of Theorem (2.1) is given in Appendix 1, Theorem (3.1) in Appendix 2 and Theorem (2.2) and Theorem(3.2) are proved in Appendix 3.

\section{A.1 Appendix 1}

We prove results for equation (2); similar arguments apply to equation (4). Some of part (b) of Theorem (2.1) holds for equation (4) only and so we prove that part only for equation (4).

Before proving the main result, we prove the following lemmas for equation (2). The first is standard so we omit its proof.

Lemma 1 . The nonnegative cone $\mathbb{R}_{+}^{3}$ is positively invariant for equation (2).

Proof. In $\Omega_{3}$, we have the following inequalities:

$$
\begin{aligned}
& S^{\prime}=d_{S} S^{0} \geq 0 \quad \text { when } S=0 \quad A^{\prime}=d_{A} A_{0}(t) \geq 0 \quad \text { when } S=0 \quad u^{\prime}=0 \geq 0 \\
& \text { when } u=0 .
\end{aligned}
$$

So the nonnegative ( $S, A, u)$-cone $\Omega_{3}$ is positively invariant for the system (2).

Lemma 2. All nonnegative solutions of equation (2) are ultimately uniformly bounded in forward time and thus they exist for all positive time. Moreover, the system (2) is dissipative in $\mathbb{R}_{+}^{3}$.

Proof. Multiplying the first equation of (2) by $\gamma$ and adding to third equation, using a new variable $z=\gamma S+u$, we arrive at the differential inequality:

$$
z^{\prime}=\left(d_{S} \gamma S^{0}-d_{S} \gamma S-d_{u} u\right)-g(S, A) u \leq d_{S} \gamma S^{0}-d(S+u)
$$

where $d=\min \left\{d_{S}, d_{u}\right\} \leq d_{S} \gamma S^{0}-d z$.

This implies

$$
z(t) \leq\left(z(0)-\gamma \frac{S^{0} d_{S}}{d}\right) e^{-d t}+\gamma \frac{S^{0} d_{S}}{d} .
$$


Thus for any initial condition in $\mathbb{R}_{+}^{3}$

$$
\limsup _{t \rightarrow \infty}(t) \leq \limsup _{t \rightarrow \infty}\left(\left(z(0)-\gamma \frac{S^{0} d_{S}}{d}\right) e^{-d t}+\gamma \frac{S^{0} d_{S}}{d}\right) \leq \gamma \frac{S^{0} d_{S}}{d}
$$

Now, from the second equation we have

$$
A^{\prime} \leq d_{A}\left(A_{0}(t)-A\right)
$$

which implies that $A$ is ultimately bounded by $M_{1}=\max A_{0}(t)$.

These inequalities prove that equation (2) is dissipative.

For a real-valued function $p$ on $[0, \infty)$, we define

$$
p_{\infty}=\liminf _{t \rightarrow \infty} p(t), \quad p^{\infty}=\limsup _{t \rightarrow \infty} p(t)
$$

Lemma 3. There exist a constant $\eta>0$, independent of initial data, such that $S_{\infty} \geq \eta$ for all solutions $S(t)$, where $\eta$ is a unique, nonzero, root of the equation $f(\eta) S^{0}+d\left(\eta-S^{0}\right)=0$.

Proof. The equation $G(v)=f(v) S^{0}+d\left(v-S^{0}\right)$ is an increasing function of $v$ satisfying $G(0)<0$ and $G\left(S^{0}\right)>0$. So, the intermediate value theorem gives the unique value of $\bar{\eta} \in\left(0, S^{0}\right)$ such that $G(\bar{\eta})=0$. Now, suppose that $S_{\infty}<\eta$. Since $u^{\infty} \leq \gamma\left(S^{0} d_{S} / d\right)$, applying Corollary 2.4(a) of Ref. [35] to the $S$ equation of (2),

$$
\begin{aligned}
0 & \geq d_{S}\left(S^{0}-S_{\infty}\right)+\gamma^{-1} f\left(S_{\infty}\right) \liminf _{t \rightarrow \infty}[-u(t)] \\
& \geq d_{S}\left(S^{0}-S_{\infty}\right)-\gamma^{-1} f\left(S_{\infty}\right) \limsup _{t \rightarrow \infty}[u(t)] \\
& \geq d_{S}\left(S^{0}-S_{\infty}\right)-f\left(S_{\infty}\right) S^{0} \frac{d_{S}}{d}>d\left(S^{0}-\eta\right)-f(\eta) S^{0}
\end{aligned}
$$

and so $f(\eta) S^{0}+d \eta>\mathrm{d} S^{0}$ which gives a contradiction due to the choice of $\eta$.

Note that for $f(S)=m(S /(a+S))$, the value of $\eta$ is,

$$
\eta=\frac{-\left(d a+m S^{0}-d S^{0}\right)+\sqrt{\left.\left(d a+m S^{0}-d S^{0}\right)^{2}+4 d^{2} S^{0} a\right)}}{2 a} .
$$

Lemma 4. There is a constant $\theta>0$, independent of initial data, such that $A_{\infty} \geq \theta$ for all solutions $A(t)$, where $\theta$ is a unique, nonzero, root of the equation $d_{S} \gamma S^{0} p(\theta)+d d_{A} \theta-$ $d d_{A} a_{1}=0$ where $\min _{t} A_{0}(t)=a_{1}$.

Proof. For any fixed $a_{1}$, the equation $F(w)=d d_{A} w+d_{S} \gamma S^{0} p(w)-d d_{A} a_{1}$ is an increasing function of $w$, satisfying $F(0)<0$ and $F\left(a_{1}\right)>0$. So by the intermediate value theorem, 
there exists a unique root $\bar{\theta} \in\left(0, a_{1}\right)$ of $F(w)$. Now, suppose that $A_{\infty}(t)<\theta$. Since $u^{\infty} \leq \gamma\left(S^{0} d_{S} / d\right)$, applying Corollary 2.4(a) of Ref. [35] to the $A$ equation of (2),

$$
\begin{aligned}
0 & \geq d_{A}\left(a_{1}-A_{\infty}\right)+p\left(A_{\infty}\right) \liminf _{t \rightarrow \infty}[-u(t)] \\
& \geq d_{A}\left(a_{1}-A_{\infty}\right)-p\left(A_{\infty}\right) \limsup _{t \rightarrow \infty}[u(t)] \\
& \geq d d_{A}\left(a_{1}-A_{\infty}\right)-\gamma S^{0} p\left(A_{\infty}\right) d_{S} \\
& >d d_{A}\left(a_{1}-\theta\right)-\gamma S^{0} p(\theta) d_{S} .
\end{aligned}
$$

This implies that $d d_{A} \theta+\gamma S^{0} p(\theta) d_{S}>d d_{A} a_{1}$ which gives a contradiction due to the choice of $\theta$.

Proof of Theorem (2.1). (a) The local stability of $E_{0}(t)$ can be determined by the Floquet exponents of the variational equation. The variational equation corresponding to $E_{0}(t)$ of equation (2) is:

$$
Z^{\prime}=\left(\begin{array}{ccc}
-d_{S} & 0 & -\gamma^{-1} f\left(S^{0}\right) \\
0 & -d_{A} & -p\left(A^{*}(t)\right) \\
0 & 0 & f\left(S^{0}\right)-d_{u}-g\left(S^{0}, A^{*}(t)\right)
\end{array}\right) Z .
$$

A computation yields the fundamental matrix $\Phi(t)$

$$
\Phi(t)=\left(\begin{array}{ccc}
e^{-d_{S} t} & 0 & \\
0 & e^{-d_{A} t} & \cdot \\
0 & 0 & e^{\int_{0}^{t}\left(f\left(S^{0}\right)-d_{u}-g\left(S^{0}, A^{*}(s)\right)\right) \mathrm{d} s}
\end{array}\right) .
$$

Note that the entries denoting "." play no role in the stability of $E_{0}(t)$. Evaluating $\Phi(t)$ at $t=T$, we obtain the multipliers $e^{-d_{S} T}, e^{-d_{A} T}$ and $e^{T\left(f\left(S^{0}\right)-d_{u}-\left[g\left(S^{0}, A^{*}(t)\right)\right]_{m}\right)}$. Then it follows immediately that $\lambda_{1}=-d_{S}, \lambda_{2}=-d_{A}$ and $\lambda=f\left(S^{0}\right)-d_{u}-\left[g\left(S^{0}, A^{*}(t)\right)\right]_{m}$ are the Floquet exponents. Two of the exponents, $\lambda_{1}, \lambda_{2}$ are automatically negative and the third, $\lambda$ is negative if $f\left(S^{0}\right)-d_{u}-\left[g\left(S^{0}, A^{*}(t)\right)\right]_{m}<0$. Thus $E_{0}(t)$ is asymptotically stable if $\lambda<0$ and is unstable if $\lambda>0$.

In order to prove global stability, we first consider the case when $S(t) \geq S^{0}$ for all $t \in^{\mathbb{R}+}$. The $S$ equation implies that

$$
S^{\prime} \leq 0
$$

i.e. $S(t)$ is non-increasing for all $t \geq 0$. Since $S$ is bounded from below, so it approaches to a limit, denote $\lim _{t \rightarrow \infty} S(t)=L \geq S^{0}$, then $\lim _{t \rightarrow \infty} S^{\prime}(t)=0$ because the $\lim _{t \rightarrow \infty} S(t)$ exists. Then $\lim _{t \rightarrow \infty} u(t)$ exists and $\lim _{t \rightarrow \infty} u(t)=\left(\gamma d_{S}\left(S^{0}-L\right) / f(L)\right)$. The positivity of $u$ implies that $L \leq S^{0}$. So the $\lim _{t \rightarrow \infty} S(t)=S^{0}$ and $\lim _{t \rightarrow \infty} u(t)=0$.

Now, consider $S(t) \leq S^{0}$ for some $t$. By positive invariance of $\left[0, S^{0}\right]$ under $S^{\prime}=d_{S}\left(S^{0}-S\right)$, we have that $S(t) \leq S^{0}$ for all large $t$. Since $\lambda<0$, we can choose $\varepsilon>0$ small enough such that $\lambda_{0}=f\left(S^{0}\right)-d_{u}-\left[g\left(S^{0}, A^{*}(t)+\varepsilon\right)\right]_{m}<0$. Then from the equation $A^{\prime}=d_{A}\left(A_{0}(t)-A\right)$, we conclude that there exists a $t_{0}$ such that $A(t) \leq A^{*}(t)+\varepsilon$ for $t>t_{0}$ 
and where $\varepsilon>0$ is chosen above. From the $u$ equation of (2), we have

$$
u((n+1) T) \leq u(n T) e^{\int_{n T}^{(n+1) T}\left(f(S(s))-d_{u}-g\left(S(s), A^{*}(s)+\varepsilon\right)\right) \mathrm{d} s}=u(n T) \lambda_{n}
$$

where

$$
\lambda_{n}=e^{\int_{n T}^{(n+1) T}\left(f(S(s))-d_{u}-g\left(S(s), A^{*}(s)+\varepsilon\right)\right) \mathrm{d} s} .
$$

This implies that

$$
\lambda_{n} \leq e^{\int_{n T}^{(n+1) T}\left(f\left(S^{0}\right)-d_{u}-g\left(S^{0}, A^{*}(s)+\varepsilon\right)\right) \mathrm{d} s}=e^{\left(f\left(S^{0}\right)-d_{u}-\left[g\left(S^{0}, A^{*}(t)+\varepsilon\right)\right]_{m}\right) T}=e^{\lambda_{0} T}
$$

and this implies that $\lim _{n \rightarrow \infty} u(n T)=0$.

In order to show that $\lim _{t \rightarrow \infty} S(t)=S^{0}$ we use Corollary 2.4(a) of Ref. [35]. From the inequality $S^{\prime} \leq d_{S}\left(S^{0}-S\right)$, we conclude that $S^{\infty} \leq S^{0}$. Let $\varepsilon>0$ and make $t$ large so that $u^{\infty}<\operatorname{rac} \varepsilon d_{S} \gamma f\left(S^{0}\right)$ (because $\lim _{t \rightarrow \infty} u(t)=0$ ). Applying Corollary 2.4(a) of Ref. [35] to the $S$ equation in (2),

$$
\begin{aligned}
0 & \geq d_{S} S^{0}-d_{S} S_{\infty}+\gamma^{-1} \liminf _{t \rightarrow \infty}[-u(t)] f\left(S_{\infty}\right) \\
& \geq d_{S} S^{0}-d_{S} S_{\infty}-\gamma^{-1} \limsup _{t \rightarrow \infty}[u(t)] f\left(S^{0}\right) \geq d_{S} S^{0}-d_{S} S_{\infty}-\varepsilon d_{S}
\end{aligned}
$$

and hence we have $S^{0} \geq S^{\infty} \geq S_{\infty} \geq S^{0}-\varepsilon$, which is true for any $\varepsilon>0$. This implies that for $t$ large enough we have $\lim _{t \rightarrow \infty} S(t)=S^{0}$. This implies that $E_{0}(t)$ is globally asymptotically stable for equation (2).

Part (b) We apply Theorem (4.1) of Ref. [16]. Using the notation, we set

$$
\begin{aligned}
& X=\left\{(S, u, A) \in \mathbb{R}_{+}^{3}: \gamma S+u+A \leq \gamma \frac{S^{0} d_{S}}{d}+M_{1},\right. \\
&\text { where } \left.M_{1}=\max _{0 \leq t \leq T}\left|A_{0}(t)\right| \text { and } d=\min \left\{d_{S}, d_{u}\right\}\right\}, \\
& X_{1}=\{(S, u, A) \in X: u \neq 0\} \text { and } X_{2}=\{(S, u, A) \in X: u=0\} .
\end{aligned}
$$

Define a map $P$ such as $P(S(0), A(0), u(0))=(S(T), A(T), u(T))$. We want to show that there exists $\varepsilon>0$ such that

$$
\liminf _{n \rightarrow \infty} d\left(P^{n}(X), X_{2}\right)>\varepsilon
$$

Given that

1. $X$ is compact metric space.

2. $P: X \rightarrow X$ is continuous map.

3. $P\left(X_{1}\right) \subset X_{1}$

4. $M$ is the maximal compact invariant set in $X_{2}$.

In our case $M=E_{0}(0)$, since the omega limit set of solutions starting in $X_{2}$ is $E_{0}(0)$ where $E_{0}(0)=\left(S^{0}, A^{*}(0), 0\right)$. We want to show that 
1. $M$ is isolated in $X$

2. $W^{s}(M) \subset X_{2}$

In order to show that $M$ is isolated in $X$ we will apply the following Theorem 2.3 of Ref. [23]. Let $V$ be the neighborhood given by this theorem. Assume that there exists an invariant set $\tilde{K}$ such that

$$
M \subset \tilde{K} \subseteq V \cap X
$$

Since $\tilde{K}$ is positively invariant, all solutions that begin in $\tilde{K}$ stay in $\tilde{K}$ and so in $V$ for positive time. Thus $\tilde{K} \subset W^{s}\left(E_{1}\right)$.

Since $\tilde{K}$ is negatively invariant, all solutions that begin in $\tilde{K}$ stay in $\tilde{K}$ and so in $V$ for negative time. Thus $\tilde{K} \subset W^{u}\left(E_{1}\right)$.

$$
W^{s}\left(E_{1}\right) \cap W^{u}\left(E_{1}\right)=E_{1}
$$

thus $\tilde{K}=M=E_{1}$. Therefore $K$ is an isolated compact invariant set in $X$.

It is clear that in this case $W^{s}(M)=X_{2}$.

For the existence of a positive periodic solution, we assume that $X_{1}$ is a convex and relatively open subset in $X$.The map $P$ satisfies the following conditions

1. $P: X \rightarrow X$ is point dissipative (since all the positive trajectories eventually lie in a bounded set);

2. $P$ is compact (since $P$ is continuous in $\mathbb{R}^{+3}$ ); and

3. $P$ is uniformly persistent with respect to $\left(X_{1}, X_{2}\right)$.

The existence of a positive $T$ periodic solution follows directly by Theorem 1.3.6 of Ref. [39].

For equation (4), it follows from Theorem 4.2 of Ref. [30], that every solution with $u(0)>0$ converges to one of these states.

If $p(A)=0$ and $d_{S}=d_{A}=d$, then the limiting system

$$
\begin{aligned}
& u^{\prime}=\left(f\left(S, A^{*}\right)-d\right) u=g_{1}(t, u) \\
& S=\frac{S^{0}}{d}-\gamma^{-1} u
\end{aligned}
$$

has concave nonlinearities. By Theorem 3.1 of Ref. [31] and Lemma 2.7 of Ref. [11], if $\lambda>0$, every solution with $u(0)>0$ converges to $E_{u}(t)$.

If $A_{0}$ is constant, $p=0$ and $\lambda>0$. Then, a unique $E_{u}$ state can be obtained by setting the right side of equation (2) equal to zero and then solving for a none-zero $S, A$ and $u$. Then $F(S)=f(S)-d_{u}-g\left(S, A_{0}\right)$, by intermediate value theorem, gives a unique $\bar{S} \in\left(0, S^{0}\right)$, because $F(0)<0$ and $F\left(S^{0}\right)>0$ such that $F(\bar{S})=0$. Also, $G(u)=\left(d_{S} S^{0}-d_{S} \bar{S}\right)-$ $u \gamma^{-1} f(\bar{S})=0$ gives a unique $\bar{u}$ such that $G(\bar{u})=0$. Thus in this case, $E_{u}$ is unique. If $\lambda<0$, then there does not exist an $\bar{S}$ such that $F(\bar{S})=0$. The local stability of $E_{u}$ is proved in the next theorem. For the global stability of $E_{u}$, we consider the limiting system

$$
\begin{aligned}
& S^{\prime}=d_{S}\left(S^{0}-S\right)-\gamma^{-1} f(S) u \\
& u^{\prime}=\left(f(S)-d_{u}-g\left(S, A^{*}\right)\right) u .
\end{aligned}
$$


We apply the Dulac criterion with the auxiliary function $g(S, u)=(1 / u)$ to equation $(15)$ and find that

$$
\left.\left.\frac{\partial}{\partial S}\left[g(S, u) S^{\prime}\right)\right]+\frac{\partial}{\partial u}\left[g(S, u) u^{\prime}\right)\right]=-\frac{d_{S}}{u}-\gamma^{-1} f^{\prime}(S)<0 .
$$

Hence the above system (15) does not have any periodic solution. This together with Poincare-Bendixson theorem implies the global stability of equation (15). For the global stability of equation (2), we use the Theorem (F.1) of Ref. [30]. Since all the hypotheses of the Theorem (F.1) are satisfied, see Ref. [18], so we conclude that $E_{u}$ is globally asymptotically stable.

\section{A.2 Appendix 2}

It is easy to see that all the lemmas of Appendix 1 also hold for equations (8) and (10). The upper bound of the solutions for equation (8) as given in Lemma (2) is

$$
\limsup _{t \rightarrow \infty}\left(\gamma S(t)+u(t)+u_{+}(t)+A(t)\right) \leq \gamma \frac{S^{0} d_{S}}{d}+M_{1},
$$

where $M_{1}=\max _{t}\left|A_{0}(t)\right|, d=\min \left\{d_{S}, d_{u}\right\}$.

All the results that are proved only for equation (8) can also be proved for equation (10) in exactly the same way. So we proved them only for equation (8).

Proof of Theorem 3.1. (a) The local stability of $E_{0}(t)$ can be determined by the Floquet exponents of the variational equation. The variational equation corresponding to $E_{0}(t)$ of equation (8) is:

$$
Z^{\prime}=\left(\begin{array}{cccc}
-d_{S} & 0 & -\gamma^{-1} f\left(S^{0}\right) & -\gamma^{-1} f\left(S^{0}\right) \\
0 & -d_{A} & -g_{1}\left(A^{*}(t)\right) & 0 \\
0 & 0 & f\left(S^{0}\right)-d_{u}-g\left(S^{0}, A^{*}(t)\right) & q f_{+}\left(S^{0}\right) \\
0 & 0 & 0 & z_{44}
\end{array}\right) Z
$$

where $z_{44}=f_{+}\left(S^{0}\right)(1-q)-d_{u}-g_{+}\left(S^{0}, A^{*}(t)\right)$. Note that the entries denoting "." play no role in the stability of $E_{0}(t)$. A computation yields the fundamental matrix $\Phi(t)$

$$
\Phi(t)=\left(\begin{array}{cccc}
e^{-d_{S} t} & 0 & \cdot & \cdot \\
0 & e^{-d_{A} t} & \cdot & 0 \\
0 & 0 & e^{\int_{0}^{t}\left(f\left(S^{0}\right)-d_{u}-g\left(S^{0}, A^{*}(s)\right)\right) d s} & \cdot \\
0 & 0 & 0 & e^{\int_{0}^{t}\left(f_{+}\left(S^{0}\right)(1-q)-d_{u}-g_{+}\left(S^{0}, A^{*}(s)\right)\right) d s}
\end{array}\right)
$$

Evaluating $\Phi(t)$ at $t=T$, we obtain the multipliers $e^{-d_{S} T}, e^{-d_{A} T}, e^{T\left(f\left(S^{0}\right)-d_{u}-\left[g\left(S^{0}, A^{*}(t)\right)\right]_{m}\right)}$ and $e^{T\left(f_{+}\left(S^{0}\right)(1-q)-d_{u}-\left[g_{+}\left(S^{0}, A^{*}(t)\right)\right]_{m}\right)}$. Then it follows immediately that $\lambda_{1}=-d_{S}, \lambda_{2}=-d_{A}, \lambda=$ $f\left(S^{0}\right)-d_{u}-\left[g\left(S^{0}, A^{*}(t)\right)\right]_{m}$ and $\lambda_{+}=f_{+}\left(S^{0}\right)(1-q)-d_{u}-\left[g_{+}\left(S^{0}, A^{*}(t)\right)\right]_{m}$ are Floquet exponents. Two of the exponents, $\lambda_{1}, \lambda_{2}$ are automatically negative and $\lambda$ and $\lambda_{+}$are 
negative if $f\left(S^{0}\right)-d_{u}-\left[g\left(S^{0}, A^{*}(t)\right)\right]_{m}<0$ and $f_{+}\left(S^{0}\right)(1-q)-d_{u}-\left[g_{+}\left(S^{0}, A^{*}\right.\right.$ $(t))]_{m}<0$. Thus, $E_{0}(t)$ is asymptotically stable if $\lambda, \lambda_{+}$both are negative, and is unstable if either $\lambda>0$ or $\lambda_{+}>0$.

For the global stability of $E_{0}$ in the constant case, since $f\left(S^{0}\right)-d_{u}-g\left(S^{0}, A_{0}\right)<0$ and $f_{+}\left(S^{0}\right)-d_{u}-g_{+}\left(S^{0}, A_{0}\right)<0$, we choose $\varepsilon>0$ small enough so that $f\left(S^{0}+\varepsilon\right)-d_{u}-$ $g\left(S^{0}+\varepsilon, A_{0}+\varepsilon\right)<0$ and $f_{+}\left(S^{0}+\varepsilon\right)-d_{u}-g_{+}\left(S^{0}+\varepsilon, A_{0}+\varepsilon\right)<0$. From the inequalities $S^{\prime} \leq d_{S}\left(S^{0}-S\right)$ and $A^{\prime} \leq d_{A}\left(A_{0}-A\right)$, we conclude that for all large $t>0, S(t) \leq$ $S^{0}+\varepsilon$ and $A(t) \leq A_{0}+\varepsilon$, where $\varepsilon>0$ is chosen above.

From the $u$ and $u_{+}$equations of the system (8)

$$
\begin{aligned}
u^{\prime}+u_{+}= & \left(f(S)-d_{u}-g(S, A)\right) u+\left(f_{+}(S)-d_{u}-g_{+}(S, A)\right) u_{+} \\
\leq & \left(f\left(S^{0}+\varepsilon\right)-d_{u}-g\left(S^{0}+\varepsilon, A_{0}+\varepsilon\right)\right) u \\
& +\left(f_{+}\left(S^{0}+\varepsilon\right)-d_{u}-g_{+}\left(S^{0}+\varepsilon, A_{0}+\varepsilon\right)\right) u_{+} .
\end{aligned}
$$

Take $m=\min \left\{f_{+}\left(S^{0}+\varepsilon\right)-d_{u}-g_{+}\left(S^{0}+\varepsilon, A_{0}+\varepsilon\right), f\left(S^{0}+\varepsilon\right)-d_{u}-g\left(S^{0}+\varepsilon, A_{0}+\right.\right.$ $\varepsilon)\}<0$. Then

$$
u^{\prime}+u_{+} \leq m\left(u+u_{+}\right)
$$

and this implies that $\limsup _{t \rightarrow \infty}\left(u(t)+u_{+}(t)\right) \leq 0$. From the positivity of $u$ and $u_{+}$, we conclude that $\lim _{t \rightarrow \infty} u(t)=0$ and $\lim _{t \rightarrow \infty} u_{+}(t)=0$

Part (b) First we will consider the case when $\lambda_{+}>0$. We can choose $\varepsilon>0$ such that $f_{+}\left(S^{0}-\varepsilon\right)(1-q)-d_{u}-\left[g_{+}\left(S^{0}+\varepsilon, A^{*}(t)+\varepsilon\right)\right]_{m}=B_{1}>0$. From the inequality $A^{\prime}(t) \leq d_{A}\left(A_{0}(t)-A\right)$, there exists a $t_{0}>0$ such that $A(t) \leq A^{*}(t)+\varepsilon, t>t_{0}$.

Pick a solution $\left(S(t), A(t), u(t), u_{+}(t)\right)$ with $u_{+}(0)>0$. We suppose that $u$ and $u_{+}$do not uniformly weakly persist and derive a contradiction. We can assume that

$$
\begin{aligned}
& u^{\infty} \leq \frac{\varepsilon \gamma d_{S}}{4 n f\left(S^{0}\right)} \leq \varepsilon \\
& u_{+}^{\infty} \leq \frac{\varepsilon \gamma d_{S}}{4 n f_{+}\left(S^{0}\right)} \leq \varepsilon
\end{aligned}
$$

where $n=\max \{1, \gamma\}$ and $\varepsilon>0$ is chosen above. From the inequality $S^{\prime} \leq d_{S}\left(S^{0}-S\right)$, we conclude that $S^{\infty} \leq S^{0}$. We apply Corollary 2.4(a) of Ref. [35] to the $S$ equation of (2)

$$
\begin{aligned}
0 & \geq d_{S} S^{0}-d_{S} S_{\infty}+\gamma^{-1} \liminf _{t \rightarrow \infty}\left[-\left(u(t) f\left(S_{\infty}\right)+u_{+}(t) f_{+}\left(S_{\infty}\right)\right)\right] \\
& \geq d_{S} S^{0}-d_{S} S_{\infty}-\gamma^{-1} \limsup _{t \rightarrow \infty}\left[u(t) f\left(S^{0}\right)+u_{+}(t) f_{+}\left(S^{0}\right)\right] \\
& \geq d_{S} S^{0}-d_{S} S_{\infty}-\frac{\varepsilon d_{S}}{4 n}-\frac{\varepsilon d_{S}}{4 n} \\
& \geq S^{0}-S_{\infty}-\frac{\varepsilon}{4 n}-\frac{\varepsilon}{4 n} \geq-S_{\infty}+S^{0}-\frac{\varepsilon}{2 n}
\end{aligned}
$$

from this, we conclude that

$$
S^{0} \geq S^{\infty} \geq S_{\infty} \geq S^{0}-\frac{\varepsilon}{2}
$$


This implies that there exists a $t_{1}>0$ such that $S(t) \in\left[S^{0}-\varepsilon, S^{0}+\varepsilon\right]$ for $t>t_{1}$. Pick a solution $\left(S(t), A(t), u(t), u_{+}(t)\right.$ ). For a large $t>\max \left\{t_{0}, t_{1}\right\}$ (say $t_{1}$ ), we have from the $u_{+}$ equation of (8):

$$
\begin{aligned}
u_{+} & \geq\left(f_{+}\left(S^{0}-\varepsilon\right)(1-q)-d_{u}-g_{+}\left(S^{0}+\varepsilon, A^{*}(t)+\varepsilon\right)+\mu u\right) u_{+} \\
& \geq\left(f_{+}\left(S^{0}-\varepsilon\right)(1-q)-d_{u}-g_{+}\left(S^{0}+\varepsilon, A^{*}(t)+\varepsilon\right)\right) u_{+}
\end{aligned}
$$

By integrating equation (17) from $N T \geq t_{1}$ to $n T$, with $n>N$, we have

$$
u_{+}(n T) \geq u_{+}(N T) e^{B_{1}(n-N) T}, \quad \text { for } n>N
$$

which gives a contradiction to equation (16) for a large enough $t$.

Now we will consider the case when $\lambda>0$. We can pick $\varepsilon>0$ (it could be different then above) such that $f\left(S^{0}-\varepsilon\right)-d_{u}-\left[g\left(S^{0}+\varepsilon, A^{*}(t)+\varepsilon\right)\right]_{m}-\mu \varepsilon=B_{2}>0$. Also, from the inequality $A^{\prime} \leq d_{A}\left(A_{0}(t)-A\right)$, we conclude that $A(t) \leq A^{*}(t)+\delta$ for some $0<\delta \leq \varepsilon$ and for $t>t_{2}$ large enough. Assume that $u^{\infty} \leq\left(\varepsilon \gamma d_{S} / 4 n f\left(S^{0}\right)\right) \leq \varepsilon$ and $u_{+}^{\infty} \leq$ $\left(\varepsilon \gamma d_{S} / 4 n f\left(S^{0}\right)\right) \leq \varepsilon$ where $n=\max \{1, \gamma\}$. As shown above, in this case there exists a $t_{1}>0$ such that $S(t) \in\left[S^{0}-\varepsilon, S^{0}+\varepsilon\right]$ where $\varepsilon>0$ is chosen above. Pick a solution $\left(S(t), A(t), u(t), u_{+}(t)\right)$ with $u(0)>0$. For $t>t^{\prime}=\max \left\{t_{1}, t_{2}\right\}$, we have from the $u$ equation of (10):

$$
\begin{aligned}
u^{\prime} & =\left[f(S)-d_{u}-g(S, A(t))\right] u+q f_{+}(S) u_{+}-\mu u u_{+} \\
& \geq\left[f\left(S^{0}-\varepsilon\right)-d_{u}-g\left(S^{0}+\varepsilon, A^{*}(t)+\varepsilon\right)-\mu \varepsilon\right] u .
\end{aligned}
$$

Integrate equation (18) from $N T \geq t^{\prime}$ to $n T$ we have:

$$
u(n T) \geq u(N T) e^{B_{2}(n-N) T}, \quad \text { for } n>N
$$

which gives a contradiction to $u^{\infty}<\varepsilon$ for large enough $n$. This shows that, if $\lambda>0$ or $\lambda_{+}>0$ then, there exists a constant $\varepsilon>0$ independent of initial data such that

$$
\limsup _{t \rightarrow \infty}\left(u(t)+u_{+}(t)\right)>\varepsilon
$$

for all solutions.

Now, we will prove the uniformly strong persistence of either $u$ or $u_{+}$. Using the notation, we set

$$
\begin{aligned}
& X=\left\{\left(S, A, u, u_{+}\right) \in \mathbb{R}_{+}^{4}: 0 \leq \gamma S+u+u_{+} A \leq \gamma \frac{S^{0} d_{S}}{d}+M_{1}, \text { where } M_{1}=\max _{0 \leq t \leq T}\left|A_{0}(t)\right|,\right. \\
& \left.d=\min \left\{d_{S}, d_{u}\right\}\right\}, \quad X_{2}=\left\{\left(S, A, u, u_{+}\right) \in X: u+u_{+}=0\right\} \\
& \text { and } \quad X_{1}=\left\{\left(S, A, u, u_{+}\right) \in X: u \neq 0 \text { or } u_{+} \neq 0\right\} .
\end{aligned}
$$


Define a map $P$ such that

$$
P\left(S(0), A(0), u(0), u_{+}(0)\right)=\left(S(T), A(T), u(T), u_{+}(T)\right) .
$$

This map $P$ satisfies the following

1. $P: X \rightarrow X$ is continuous map;

2. $P\left(X_{1}\right) \subset X_{1}$

3. $P$ has a global attractor.

(iii) holds for $P$ because the map $P$ is compact and point dissipative. By Theorem 1.3.3. of Ref. [39], there exists an $\varepsilon>0$ independent of initial data such that $\liminf _{n \rightarrow \infty}\left(u(n T)+u_{+}(n T)\right)>\varepsilon$. From this, we conclude that

$$
\liminf _{t \rightarrow \infty}\left(u(t)+u_{+}(t)\right)>\varepsilon
$$

for all solutions with $u(0)>0, u_{+}(0)>0$.

Now, we will show that if $u_{+\infty}>\varepsilon$ for some $\varepsilon>0$, then there exists some $\varepsilon_{1}>0$ such that $u^{\infty}>\varepsilon_{1}$. Since $A^{\infty} \leq M_{1}=\max _{0 \leq t \leq T} A^{*}(t)$, we apply Corollary 2.4(a) of Ref. [35] to the $u$ equation in (8)

$$
\begin{aligned}
0 \geq & \liminf _{t \rightarrow \infty}\left[\left(f(S(t))-d_{u}-g(S(t), A(t))\right) u_{\infty}+q f_{+}(S(t)) u_{+}(t)-\mu u_{\infty} u_{+}(t)\right] \\
\geq & \liminf _{t \rightarrow \infty}\left[\left(f(S(t))-d_{u}-g(S(t), A(t))\right)\right] u_{\infty}+\liminf _{t \rightarrow \infty}\left(q f_{+}(S(t)) u_{+}(t)\right) \\
& -\mu u_{\infty} \limsup _{t \rightarrow \infty} u_{+}(t) \\
\geq & \left(f(\eta)-d_{u}-g\left(S^{0}, M_{1}\right)\right) u_{\infty}+q f_{+}(\eta) u_{+\infty}-\mu u_{\infty} \gamma \frac{S^{0} d_{S}}{d} .
\end{aligned}
$$

Here we have used that $\liminf _{t \rightarrow \infty} S(t) \geq \eta$, by the Lemma (3). Solving for $u^{\infty}$, we obtain

$$
u_{\infty}>\frac{q f_{+}(\eta) \varepsilon}{\left(-f(\eta)+d_{u}+g\left(S^{0}, M_{1}\right)+\mu \gamma \frac{S^{0} d_{S}}{d}\right)}=\varepsilon_{1}
$$

which is positive by Lemma (3), independent of the initial data. This implies that if $u_{+}$ persists then so does $u$.

The existence of at least one non-zero, positive, periodic solution of form $E_{u}(t)$ or $E_{u^{*}}(t)$ follows by Theorem 1.3.6 of Ref. [39] as shown in the previous theorem.

Now suppose that $\lambda<0, \lambda_{+}>0$ and $p=0$. It is easy to see that if $S(t) \geq S^{0}$ for all $t \geq 0$, then in this case $u_{+}$uniformly persists.

Consider $S(t) \leq S^{0}$ for large $t$. Since $\lambda_{+}>0$, there exists some $\varepsilon_{0}>0$ such that either $u^{\infty}>\varepsilon_{0}$ or $u_{+}^{\infty}>\varepsilon_{0}$. Since $\lambda<0$, we can pick some $\delta>0$ small enough so that $f\left(S^{0}\right)-d_{u}-\left[g\left(S^{0}, A^{*}(t)-\delta\right)\right]_{m}<0$. From the fact that $\lim _{t \rightarrow \infty} A(t)=A^{*}(t)$, we have $A^{*}(t)-\delta \leq A(t) \leq A^{*}(t)+\delta$ where $\delta>0$ is chosen above for $t$ large enough. As $f(S)-$ $d_{u}-g\left(S, A^{*}\right)$ is nondecreasing in $S \in\left[0, S^{0}\right]$ for each fixed $t \in[0, T]$, the $u$ equation of (8) 
can be written as

$$
\begin{aligned}
& u^{\prime}(t) \leq\left[f\left(S^{0}\right)-d_{u}-g\left(S^{0}, A\right)\right] u+q f_{+}\left(S^{0}\right) u_{+}-\mu u u_{+} \\
& u^{\prime}(t) \leq\left[f\left(S^{0}\right)-d_{u}-g\left(S^{0}, A^{*}(t)-\delta\right)\right] u+q f_{+}\left(S^{0}\right) u_{+} .
\end{aligned}
$$

This implies, by using the comparison theorem and the well known result from Ref. [9], that there exists a constant $e>0$ such that $u^{\infty} \leq e \lim \sup _{t \rightarrow \infty}\left|q f_{+}\left(S^{0}\right) u_{+}(t)\right|$. Next, we suppose that the $u_{+}$is not uniformly persistant and derive a contradiction. Choose some $0<\varepsilon<\varepsilon_{0}$ such that $u_{+}^{\infty}<\min \left\{\varepsilon,\left(\varepsilon / e q f_{+}\left(S^{0}\right)\right)\right\}$ where $e$ is defined above. Then, from $u^{\infty} \leq e \lim \sup _{t \rightarrow \infty}\left(q f_{+}\left(S^{0}\right) u_{+}(t)\right)$, we have $u^{\infty} \leq \varepsilon<\varepsilon_{0}$, in contradiction to the fact that $u^{\infty}>\varepsilon$. Thus $u_{+}^{\infty}>\varepsilon$ for some $\varepsilon>0$.

Now we will prove that $u_{+\infty}>\varepsilon$ for some $\varepsilon>0$. Using the notation, we set

$$
\begin{aligned}
& X=\left\{\left(S, A, u, u_{+}\right) \in \mathbb{R}_{+}^{4}: 0 \leq \gamma S+u+u_{+} A \leq \gamma \frac{S^{0} d_{S}}{d}+M_{1},\right. \\
& \text { where } \left.M_{1}=\max _{0 \leq t \leq T}\left|A_{0}(t)\right|, d=\min \left\{d_{S}, d_{u}\right\}\right\}, \\
& X_{2}=\left\{\left(S, A, u, u_{+}\right) \in X: u_{+}=0\right\} \quad \text { and } \quad X_{1}=\left\{\left(S, A, u, u_{+}\right) \in X: u_{+} \neq 0\right\} .
\end{aligned}
$$

By Theorem 1.3.3. of Ref. [39], there exists an $\varepsilon>0$ independent of initial data such that $\liminf _{n \rightarrow \infty} u_{+}(n T)>\varepsilon$ and we conclude that $\liminf _{t \rightarrow \infty} u_{+}(t)>\varepsilon$ with $\varepsilon>0$ not depending on the initial data.

The existence of one periodic solution $E_{u^{*}}(t)$ follows from Theorem 1.3.6 of Ref. [39].

Part (c) The local stability of $E_{u}=(\bar{S}, \bar{A}, \bar{u}, 0)$ is determined by the eigenvalues of the matrix

$$
C=\left(\begin{array}{cccc}
-d_{S}-\gamma^{-1} \bar{u} f(\bar{S}) & 0 & -\gamma^{-1} f(\bar{S}) & 0 \\
0 & -d_{A}-\bar{u} p^{\prime}(\bar{A}) & -p(\bar{A}) & 0 \\
\bar{u}\left(f^{\prime}(\bar{S})-g_{S}(\bar{S}, \bar{A})\right) & -\bar{u} g_{A}(\bar{S}, \bar{A}) & 0 & q f_{+}(\bar{S})-\mu \bar{u} \\
0 & 0 & 0 & c_{44}
\end{array}\right)
$$

where $c_{44}=f_{+}(\bar{S})(1-q)-d_{u}-g_{+}(\bar{S}, \bar{A})-\mu \bar{u}, \quad c_{33}=f(\bar{S})-d_{u}-g(\bar{S}, \bar{A})=0, g_{S}(\bar{S}, \bar{A})$ and $g_{A}(\bar{S}, \bar{A})$ are the derivatives of $g(S, A)$ with respect to $S$ and $A$ at $(\bar{S}, \bar{A})$, respectively. Here we have used that $c_{33}=0$ at $E_{u}$ steady state. Note that in this case $\bar{A}=A_{0}$. The eigenvalues of the matrix $C$ are the eigenvalue

$$
\bar{\lambda}=f_{+}(\bar{S})(1-q)-d_{u}-g_{+}(\bar{S}, \bar{A})+\mu \bar{u}
$$


and the eigenvalues of the matrix

$$
C_{1}=\left(\begin{array}{ccc}
-d_{S}-\gamma^{-1} \bar{u} f^{\prime}(\bar{S}) & 0 & -\gamma^{-1} f(\bar{S}) \\
0 & -d_{A}-\bar{u} p^{\prime}(\bar{A}) & -p(\bar{A}) \\
\left(f^{\prime}(\bar{S})-g_{S}(\bar{S}, \bar{A})\right) \bar{u} & -\bar{u} g_{A}(\bar{S}, \bar{A}) & 0
\end{array}\right)
$$

We compute the coefficients of the characteristic polynomial $P$ of the matrix of $C_{1}$. Let $P(\lambda)=\lambda^{3}+a_{1} \lambda^{2}+a_{2} \lambda+a_{3}$. Then

$$
a_{1}=-\operatorname{Tr}\left(C_{1}\right)=d_{S}+\gamma^{-1} \bar{u} f(\bar{S})+d_{A}+\bar{u} p^{\prime}(\bar{A})>0,
$$

and

$$
\begin{aligned}
a_{3} & =-\operatorname{Det}\left(C_{1}\right) \\
& =-\left(d_{S}+\gamma^{-1} \bar{u} f(\bar{S})\right) p(\bar{A}) \bar{u} g_{A}(\bar{S}, \bar{A})+\left(d_{A}+\bar{u} p^{\prime}(\bar{A})\right)\left(f^{\prime}(\bar{S})-g_{S}(\bar{S}, \bar{A})\right) \gamma^{-1} \bar{u} f(\bar{S})
\end{aligned}
$$

and

$$
a_{2}=\left(d_{S}+\gamma^{-1} \bar{u} f(\bar{S})\right)\left(d_{A}+\bar{u} p^{\prime}(\bar{A})\right)+\left(f^{\prime}(\bar{S})-g_{S}(\bar{S}, \bar{A})\right) \gamma^{-1} \bar{u} f(\bar{S})-p(\bar{A}) \bar{u} g_{A}(\bar{S}, \bar{A})
$$

Now, if $p(A)=0$, then $a_{3}=d_{A}\left(f^{\prime}(\bar{S})-g_{S}(\bar{S}, \bar{A})\right) \gamma^{-1} \bar{u} f(\bar{S})>0$ and

$$
\begin{aligned}
s= & a_{1} a_{2}-a_{3} \\
= & \left(d_{S}+d_{A}+\gamma^{-1} \bar{u} f(\bar{S})\right)\left[\left(d_{S}+\gamma^{-1} \bar{u} f(\bar{S})\right) d_{A}+\left(f^{\prime}(\bar{S})-g_{S}(\bar{S}, \bar{A})\right) \gamma^{-1} \bar{u} f(\bar{S})\right] \\
& -\left[d_{A}\left(f^{\prime}(\bar{S})-g_{S}(\bar{S}, \bar{A})\right) \gamma^{-1} \bar{u} f(\bar{S})\right] \\
= & \left(d_{S}+\gamma^{-1} \bar{u} f(\bar{S})\right)\left[d_{S}+\gamma^{-1} \bar{u} f(\bar{S}) d_{A}+\left(f^{\prime}(\bar{S})-g_{S}(\bar{S}, \bar{A})\right) \gamma^{-1} \bar{u} f(\bar{S})\right] \\
& +\left[\left(d_{S}+\gamma^{-1} \bar{u} f(\bar{S})\right) d_{A}\right] \\
= & \left(d_{S}+\gamma^{-1} \bar{u} f(\bar{S})\right)\left[d_{A}\left(d_{S}+\gamma^{-1} \bar{u} f(\bar{S})\right)+\left(d_{A}\right)^{2}+\left(f^{\prime}(\bar{S})-g_{S}(\bar{S}, \bar{A})\right) \bar{u} \gamma^{-1} f(\bar{S})\right]>0 .
\end{aligned}
$$

By the Routh-Hurwitz criterion all three eigenvalues have negative real parts. Thus, $E_{1}$ is asymptotically stable if $f_{+}(\bar{S})(1-q)-d_{u}-g_{+}(\bar{S}, \bar{A})+\mu \bar{u}<0$ and is unstable if $f_{+}(\bar{S})(1-q)-d_{u}-g_{+}(\bar{S}, \bar{A})+\mu \bar{u}>0$.

Now we will prove the persistence result for the autonomous case. We follow a similar argument used in Theorem 5.3 of Ref. [32], applying Theorem 4.6 in Ref. [35]. Using the notation of that result, we set

$$
\begin{gathered}
X=\left\{\left(S, A, u, u_{+}\right) \in \mathbb{R}_{+}^{4}: 0 \leq \gamma S+A+u+u_{+} \leq \gamma \frac{S^{0} d_{S}}{d}+A_{0}, \text { where } d=\min \left\{d_{S}, d_{u}\right\}\right\}, \\
X_{2}=\left\{\left(S, A, u, u_{+}\right) \in X: u_{+}=0\right\} \text { and } X_{1}=X \backslash X_{2} .
\end{gathered}
$$


We want to show that solutions which start in $X_{1}$ are eventually bounded away from $X_{2}$. Using the notation $x(t)=\left(S(t), A(t), u(t), u_{+}(t)\right)$ for a solution of equation (8), define

$$
Y_{2}=\left\{x(0) \in X_{2}: x(t) \in X_{2}, t \geq 0\right\}=\left\{x(0) \in X: u_{+}(0)=0\right\}
$$

and $\Omega_{2}$, the union of omega limit sets of solutions starting in $X_{2}$, is, by Theorem (2.1), the set $\left\{E_{0}, E_{u}\right\}$ where $E_{0}:=\left(S^{0}, A_{0}, 0,0\right)$ and $E_{u}:=(\bar{S}, \bar{A}, \bar{u}, 0)$. We will show that if $M_{0}=\left\{E_{0}\right\}$ and $M_{1}=\left\{E_{u}\right\}$, then $\left\{M_{0}, M_{1}\right\}$ is an isolated acyclic covering of $\Omega_{2}$ in $Y_{2}$ and each $M_{i}$ is a weak repeller. All solutions starting in $Y_{2}$ but not on the $S A$-plane converge to $E_{u}$ while those on the $S A$-plane converge to $E_{0}$. $E_{u}$, being locally asymptotically stable relative to $Y_{2}$, cannot belong to the alpha limit set of any full orbit in $X_{2}$ different from $E_{u}$ itself. Similar arguments apply to $E_{0}$; the only solutions converging to it lie on the $S A$-plane and these are either unbounded or leave $X$ in backward time. Thus $\left\{M_{0}, M_{1}\right\}$ is an acyclic covering of $\Omega_{2}$. If $M_{1}$ were not a weak repeller for $X_{1}$, there would exist an $x(0) \in X_{1}$ such that $x(t) \rightarrow E_{u}$ as $t \rightarrow \infty$. Let $V(t)=\left(u(t), u_{+}(t)\right)^{t}$ and define the matrix $P(S, A, u)$ by

$$
\left(\begin{array}{cc}
f(S)-d_{u}-g(S, A) & q f_{+}(S)-\mu u \\
0 & f_{+}(S)(1-q)-d_{u}-g_{+}(S, A)+\mu u
\end{array}\right)
$$

Then $C=P(\bar{S}, \bar{A}, \bar{u})$ and we may write the equation satisfied by $V(t)$ as

$$
\dot{V}=P(\bar{S}, \bar{A}, \bar{u}) V+[P(S, A, u)-P(\bar{S}, \bar{A}, \bar{u})] V
$$

If $P(\bar{S}, \bar{A}, \bar{u})^{t} W=q W$ where $q=s(P(\bar{S}, \bar{A}, \bar{u}))=s(C)>0$ and $W=(m, n)^{t}$ with $m, n>0$ is the Perron-Frobenius eigenvector, then on taking the scalar product of both sides of the differential equation by $W$ and using that $S(t) \rightarrow \bar{S}, A(t) \rightarrow \bar{A}$ and $u(t) \rightarrow \bar{u}$, we have

$$
\frac{\mathrm{d}}{\mathrm{d} t}\left(m u+n u_{+}\right) \geq q / 2\left(m u+n u_{+}\right)
$$

for all large $t$. But this leads to the contradiction to $x(t) \rightarrow E_{u}$, namely that $m u(t)+n u_{+}(t) \rightarrow$ $\infty$ as $t \rightarrow \infty$. Thus $M_{1}$ is a weak repeller. The argument above together with the fact that $E_{u}$ is locally asymptotically stable relative to the subspace $u_{+}=0$ implies that it is an isolated compact invariant set in $X$. Similar arguments show that $M_{0}$ is a weak repeller and an isolated compact invariant set in $X$. Therefore, Theorem 4.6 in Ref. [35] implies our result: there exists $\varepsilon>0$ such that $\liminf _{t \rightarrow \infty} d\left(x(t), X_{2}\right)>\varepsilon$ for all $x(0) \in X_{1}$, where $d\left(x, X_{2}\right)$ is the distance from $x$ to $X_{2}$.

\section{A.3 Appendix 3}

Let

$$
B_{0}=\frac{\gamma d_{S} S^{0}}{\min \left\{d_{S}, d_{u}\right\}}
$$


and let $(s, U)$ be the unique solution of

$$
\begin{aligned}
& B_{0}=u+\gamma S \\
& 0=d_{S}\left(S^{0}-S\right)-\gamma^{-1} f(S) u
\end{aligned}
$$

with $s, U>0 .(s, U)$ relates to the pretreatment equilibrium $(\bar{S}, \bar{u})$ as follows:

$$
s<\bar{S}, \bar{u}<U<B_{0}
$$

If $f(S)=(m S / a+S)$ then $U$ is the positive root of

$$
\left(m-d_{S}\right) U^{2}+\gamma\left[d_{S} a+2 d_{S} x S^{0}-d_{S} S^{0}-m x S^{0}\right] U+d_{S} \gamma^{2} S^{0}\left(a+x S^{0}\right)(1-x)
$$

and $x=\left(\gamma d_{S} / \min \left\{d_{S}, d_{u}\right\}\right)$. Due to the ugliness of this expression, the reader may wish to substitute $B_{0}$ for $U$ in the formulae to follow; the results continue to hold in this case.

Consider the $T$-periodic scalar equation

$$
A^{\prime}=d_{A}\left(A_{0}(t)-A\right)-U p(A)
$$

in which $u$ has been set to the constant value $u=U$. It has a unique $T$-periodic solution $A_{*}(t)=A_{*}(t+T)$ and it satisfies

$$
0<A_{*}(t) \leq A^{*}(t)
$$

Also, observe that

$$
p=0 \Rightarrow A_{*}(t)=A^{*}(t)
$$

Now define

$$
\lambda_{*}=\lambda_{*}\left(S^{0}\right)=f\left(S^{0}\right)-d_{u}-\left[g\left(S^{0}, A_{*}\right)\right]_{m}
$$

Observe that

$$
\lambda_{*} \geq \lambda
$$

and

$$
p=0 \Rightarrow \lambda_{*}=\lambda .
$$

First of all we will prove that if $\lambda_{*}<0$ then $u(t) \rightarrow 0$ as $t \rightarrow \infty$ for every solution of equation (2).

The following generalizes Theorem 2.1 (a):

Theorem 1. If $\lambda_{*}<0$, then $u(t) \rightarrow 0$ as $t \rightarrow \infty$ for every solution of equation (2). 
Proof. From the Lemma (2), we have

$$
\limsup _{t \rightarrow \infty} u \leq B_{0}
$$

The Lemma (3) gives

$$
\liminf _{t \rightarrow \infty} S \geq \eta
$$

It follows that

$$
\limsup _{t \rightarrow \infty} u \leq \limsup _{t \rightarrow \infty}(\gamma S+u)-\gamma \liminf _{t \rightarrow \infty} S \leq B_{0}-\gamma \eta\left(B_{0} / \gamma\right)=B_{1}
$$

Applying the Lemma (3) again, we obtain a better (bigger) lower bound for the limit inferior of $S$ :

$$
\liminf _{t \rightarrow \infty} S \geq \eta\left(B_{1}\right)
$$

where $\eta=\eta\left(B_{1}\right)$ is the unique root $S=\eta$ of $0=d_{S}\left(S^{0}-S\right)-\gamma^{-1} f(S) B_{1}$. If $F(b)=B-\gamma \eta(b / \gamma)$, then $F(0)=B_{0}$ and $F^{\prime}(b)<0$ because $\eta(b)$ is an increasing function of $b$. Moreover, $B_{0}>U=F(U)>F\left(B_{0}\right)=B_{1}$, from which we conclude that

$$
\limsup _{t \rightarrow \infty} u \leq U .
$$

Now suppose that $\lambda_{*}<0$. This implies that there exists $\varepsilon>0$ such that

$$
\mu=f\left(S^{0}\right)-d_{u}-\left[g\left(S^{0}, A_{*}-\varepsilon\right)\right]_{m}<0
$$

Let $(S, A, u)$ be a solution of equation (2). Given $\delta>0$, there exists $\tau>0$ such that

$$
u(t) \leq U+\delta, t \geq \tau
$$

Then

$$
A^{\prime} \geq d_{A}\left(A_{0}(t)-A\right)-(U+\delta) p(A), \quad t \geq \tau
$$

so $A(t) \geq A_{\delta}(t), t \geq \tau$ where $A_{\delta}(t)$ is the solution of

$$
A^{\prime}=d_{A}\left(A_{0}(t)-A\right)-(U+\delta) p(A)
$$

satisfying $A_{\delta}(\tau)=A(\tau)$. But $A_{\delta}(t) \rightarrow A_{\delta}^{*}(t)$ where the latter is the unique $T$-periodic solution of this differential equation. It is not difficult to verify that $A_{\delta}^{*}(t) \rightarrow A_{*}(t)$ as $\delta \rightarrow 0$ uniformly on compact sets so by choosing $\delta$ to be sufficiently small, there exists $\bar{\tau}>\tau$ so that

$$
A(t) \geq A_{\delta}(t) \geq A_{*}(t)-\varepsilon, \quad t \geq \bar{\tau}
$$

This implies that

$$
f(S(t))-d_{u}-g(S(t), A(t)) \leq f\left(S^{0}\right)-d_{u}-g\left(S^{0}, A_{*}(t)-\varepsilon\right), \quad t \geq \bar{\tau}
$$

Since

$$
u^{\prime} \leq u\left[f\left(S^{0}\right)-d_{u}-g\left(S^{0}, A_{*}(t)-\varepsilon\right)\right], \quad t \geq \bar{\tau}
$$


we have that

$$
u(t+T) \leq u(t) \exp (\mu T), \quad t \geq \bar{\tau}
$$

implying that if $\lambda_{*}<0$ then $u(t) \rightarrow 0$ as $t \rightarrow \infty$ for every solution of equation (2).

Proof of Theorem (2.2) and Theorem (3.2). Now, if

$$
p(A) \leq c A, \quad 0 \leq A \leq \max _{t} A_{0}(t)
$$

then $A_{*}(t) \geq \tilde{A}(t)$ where $\tilde{A}(t)$ is the unique $T$-periodic solution of the linear equation

$$
A^{\prime}=d_{A}\left(A_{0}(t)-A\right)-B_{0} c A .
$$

Observe that

$$
[\tilde{A}]_{m}=\frac{d_{A}}{d_{A}+B_{0} c}\left[A_{0}\right]_{m}
$$

By monotonicity of $g$

$$
\lambda_{*} \leq f\left(S^{0}\right)-d_{u}-\left[g\left(S^{0}, \tilde{A}\right)\right]_{m}
$$

Consequently, if $f\left(S^{0}\right)-d_{u}-\left[g\left(S^{0}, \tilde{A}\right)\right]_{m}<0$, then $\lambda_{*}<0$. If $g$ is concave in A, then $g$ is convex so by Jensen's inequality, we have

$$
\begin{aligned}
f\left(S^{0}\right)-d_{u}-\left[g\left(S^{0}, \tilde{A}\right)\right]_{m} & \leq f\left(S^{0}\right)-d_{u}-g\left(S^{0},[\tilde{A}]_{m}\right) \\
& =f\left(S^{0}\right)-d_{u}-\left[g\left(S^{0}, \frac{d_{A}}{d_{A}+B_{0} c}\left[A_{0}\right]_{m}\right)\right] .
\end{aligned}
$$

We note that

$$
\tilde{A}(t)=d_{A} \int_{0}^{T} G(t, s) A_{0}(s) \mathrm{d} s
$$

where Green's function $G(t, s)$ is given by

$$
G(t, s)=\left\{\begin{array}{cc}
\frac{\exp (-\mu(t-s) \exp (\mu T)}{\exp (\mu T)-1} & , 0 \leq s<t \leq T \\
\frac{\exp (-\mu(t-s))}{\exp (\mu T)-1} & , 0 \leq t \leq s \leq T
\end{array}\right.
$$

and $\mu=d_{A}+c U$.

Theorem (3.2) can be proved as above by adding the equations for $u$ and $u_{+}$, defining $v=u+u_{+}$and noticing that $f(S)-d_{u}-g(S, A) \leq f(S)-d_{u}-g_{+}(S, A)$ and $f_{+}(S)-d_{u}-g_{+}(S, A) \leq f(S)-d_{u}-g_{+}(S, A)$. 


\section{B Bifurcation results}

Theorem 1 . There is a family of positive solutions bifurcating from trivial solution $\left(S^{0}, A_{0}, 0\right)$ of the linearized system as parameter $k$ is varied near $k_{c}=\left(\left(f\left(S^{0}\right)-d_{u} / g\left(S^{0}, A_{0}\right)\right)\right)$. To leading order in the small parameter $\varepsilon$

$$
(S(\varepsilon), A(\varepsilon), u(\varepsilon))=\left(S^{0}, A_{0}, 0\right)+\varepsilon \zeta+O\left(\varepsilon^{2}\right) \quad k(\varepsilon)=k_{c}+\varepsilon k_{1}+O\left(\varepsilon^{2}\right)
$$

where $\zeta=\left(\left(-\gamma^{-1} f\left(S^{0}\right) / d_{S}\right),\left(-g_{1}\left(A_{0}\right) / d_{u}\right), 1\right)$ is the right eigenvector. Moreover, $\operatorname{sign}(u(\varepsilon))=\operatorname{sign}(\varepsilon)$ for small $\varepsilon$ and $k_{1}$ is

$$
k_{1}=\frac{\left(f^{\prime}\left(S^{0}\right)-k_{c} g_{S}\left(S^{0}, A_{0}\right)\right) \frac{\gamma^{-1} f\left(S^{0}\right)}{d_{S}}+k_{c} g_{A}\left(S^{0}, A_{0}\right) \frac{p\left(A_{0}\right)}{d_{A}}}{g\left(S^{0}, A_{0}\right)} .
$$

The largest real part of the eigenvalue for a bifurcating positive solution is given as:

$$
\gamma(\varepsilon)=k_{1} g\left(S^{0}, A_{0}\right) \varepsilon+O\left(\varepsilon^{2}\right) \text { for small } \varepsilon
$$

If $k_{1}<0$, then $\gamma(\varepsilon)<0$ for $\varepsilon>0$ and so the bifurcating solution is stable.

If $k_{1}>0$, then $\gamma(\varepsilon)>0$ for $\varepsilon>0$ and so the bifurcating solution is unstable.

Let us assume that $d_{S}=d_{A}$.

Proposition 1. Parameter $k_{1}<0$ so the positive solution $(S(\varepsilon), A(\varepsilon), u(\varepsilon))$ exists for $k<k_{c}$ near $k_{c}$ and is asymptotically stable for $\varepsilon>0$ in the following cases:

1. For $p(A)=0$ and $g(S, A)=f(S) p_{1}(A)$.

2. For $p(A)=C A$ and $g(S, A)=k A$ if $f^{\prime}\left(S^{0}\right)>\gamma\left(\left(f\left(S^{0}\right)-d_{u}\right) / f\left(S^{0}\right)\right) C$.

3. For any $p(A)$ and $g(S, A)=p_{1}(A)$, if $f^{\prime}\left(S^{0}\right)>\gamma\left(\left(f\left(S^{0}\right)-d_{u}\right) / f\left(S^{0}\right)\right) \times$ $\left(p_{1}\left(A_{0}\right) / p_{1}\left(A_{0}\right)\right) p\left(A_{0}\right)$.

4. For $g(S, A)=f(S) p_{1}(A)$,

$$
f^{\prime}\left(S^{0}\right)>\frac{\left(f\left(S^{0}\right)-d_{u}\right)}{f\left(S^{0}\right)}\left(f^{\prime}\left(S^{0}\right) p_{1}\left(A_{0}\right)+\gamma p_{1}\left(A_{0}\right) p\left(A_{0}\right)\right)
$$

Proposition 2. Parameter $k_{1}>0$ so the positive solution $(S(\varepsilon), A(\varepsilon), u(\varepsilon))$ exists for $k>k_{c}$ near $k_{c}$ and is unstable for $\varepsilon>0$ in the following cases:

1. For $p(A)=C A$ and $g(S, A)=k A$ if $f^{\prime}\left(S^{0}\right)<\gamma\left(\left(f\left(S^{0}\right)-d_{u}\right) / f\left(S^{0}\right)\right) C$.

2. For any $p(A)$ and $g(S, A)=p_{1}(A)$, if $f^{\prime}\left(S^{0}\right)<\gamma\left(\left(f\left(S^{0}\right)-d_{u}\right) / f\left(S^{0}\right)\right) \times$ $\left(p_{1}\left(A_{0}\right) / p_{1}\left(A_{0}\right)\right) p\left(A_{0}\right)$. 
3. For $g(S, A)=f(S) p_{1}(A)$,

$$
f^{\prime}\left(S^{0}\right)>\frac{\left(f\left(S^{0}\right)-d_{u}\right)}{f\left(S^{0}\right)}\left(f^{\prime}\left(S^{0}\right) p_{1}\left(A_{0}\right)+\gamma p_{1}\left(A_{0}\right) p\left(A_{0}\right)\right)
$$

Theorem 2 . There is a family of positive $T$ periodic solutions bifurcating from trivial periodic solution $\left(S^{0}, A^{*}(t), 0\right)$ of the above plasmid-free system as parameter $k$ is varied near $k_{c}=\left(f\left(S^{0}\right)-d_{u} /\left[g\left(S^{0}, A_{0}(t)\right)\right]_{m}\right)$. To leading order in the small parameter $\varepsilon$

$$
(S(t, \varepsilon), A(t, \varepsilon), u(t, \varepsilon))=\left(S^{0}, A^{*}(t), 0\right)+\varepsilon \zeta(t)+O\left(\varepsilon^{2}\right) \quad k(\varepsilon)=k_{c}+\varepsilon k_{1}+O\left(\varepsilon^{2}\right)
$$

where $\zeta(t)$ is the $T$ periodic solution of the variational equation about $(0,0,0)$. Moreover, $\operatorname{sign} u(t, \varepsilon)=\operatorname{sign} \varepsilon$ for small $\varepsilon$ and $k_{1}$ is

$$
k_{1}=\frac{\int_{0}^{T}\left[\left(f^{\prime}\left(S^{0}\right)-k_{c} g_{S}\left(S^{0}, A^{*}(s)\right)\right) \zeta_{1}(s)-k_{c} g_{A}\left(S^{0}, A^{*}(s)\right) \zeta_{2}(s)\right] \mathrm{d} s}{\left.\left[g\left(S^{0}, A^{*}\right)(t)\right)\right]_{m}} .
$$

The largest real part of Floquet exponent for bifurcating periodic solution is given as:

$$
\left.\gamma(\varepsilon)=k_{1}\left[g\left(S^{0}, A^{*}\right)(t)\right)\right]_{m} \varepsilon+O\left(\varepsilon^{2}\right) \text { for small } \varepsilon
$$

If $k_{1}<0$, then $\gamma(\varepsilon)<0$ for $\varepsilon>0$ and so the bifurcating solution is stable.

If $k_{1}>0$, then $\gamma(\varepsilon)>0$ for $\varepsilon>0$ and so the bifurcating solution is unstable.

Proposition 3. Parameter $k_{1}<0$ so the positive $T$ periodic solution $(S(t, \varepsilon), A(t, \varepsilon), u(t, \varepsilon))$ exists for $k<k_{c}$ near $k_{c}$ and is asymptotically stable for $\varepsilon>0$ in the following cases:

1. For $p(A)=0$ and $g(S, A)$ is a constant in $S$ and $A$.

2. For $p(A)=0$, if $\left.f^{\prime}\left(S^{0}\right)>\left(f\left(S^{0}\right)-d_{u} /\left[g\left(S^{0}, A^{*}\right)(t)\right)\right]_{m}\right)\left[g_{S}\left(S^{0}, A^{*}(t)\right]_{m}\right.$

3. For $p(A)=C A$, if

$$
f^{\prime}\left(S^{0}\right)>\frac{\left(f\left(S^{0}\right)-d_{u}\right)\left[g_{S}\left(S^{0}, A^{*}(t)\right)\right]_{m}}{\left.\left[g\left(S^{0}, A^{*}\right)(t)\right)\right]}+\frac{\gamma\left(f\left(S^{0}\right)-d_{u}\right)\left[g_{A}\left(S^{0}, A^{*}(t)\right)\right]_{m} C\left[A^{*}(t)\right]_{m}}{\left.\left[g\left(S^{0}, A^{*}\right)(t)\right)\right]}
$$

Theorem 3. There is $K \geq k_{c}$ such that for each $k \in[0, K)$, there exists a nontrivial periodic solution $(S, A, u, k) \in B(T) \times R$ of equation (2) satisfying $0<S<S^{0}, 0<A<A^{*}, 0<u$ for all $t$.

For the proof of these results see Ref. [19]. 


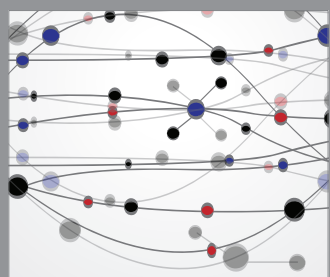

The Scientific World Journal
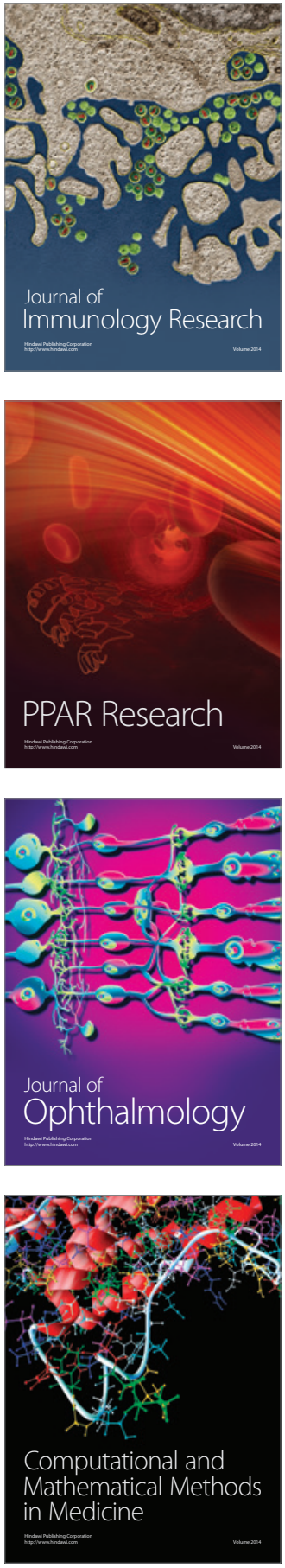

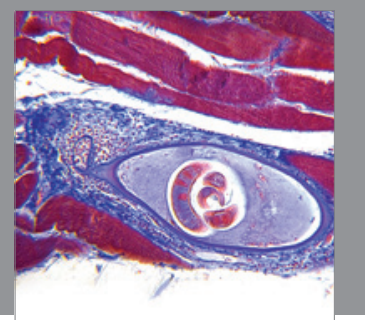

Gastroenterology

Research and Practice
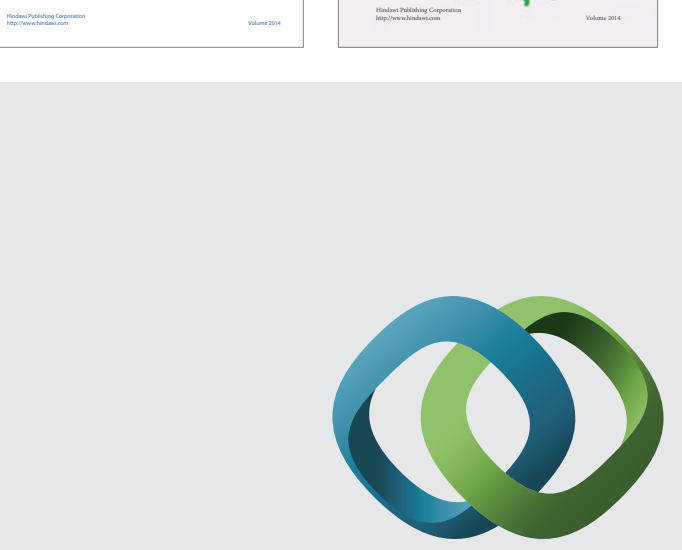

\section{Hindawi}

Submit your manuscripts at

http://www.hindawi.com
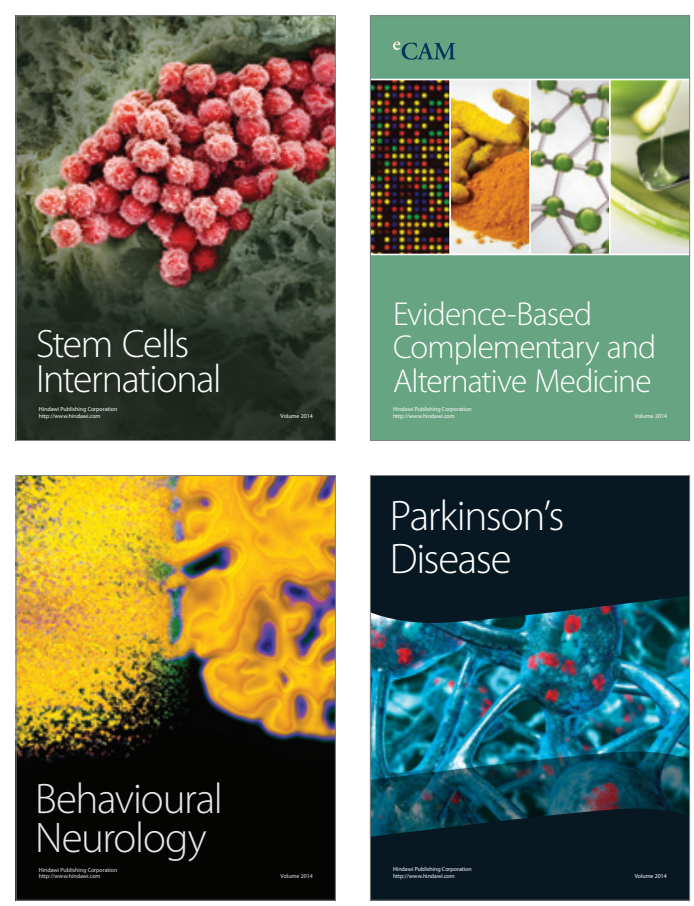

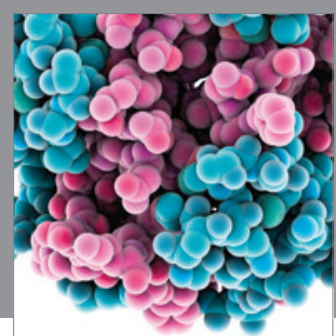

Journal of
Diabetes Research

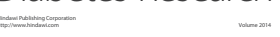

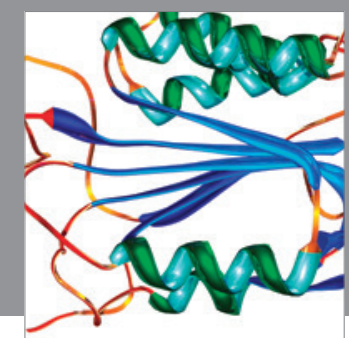

Disease Markers
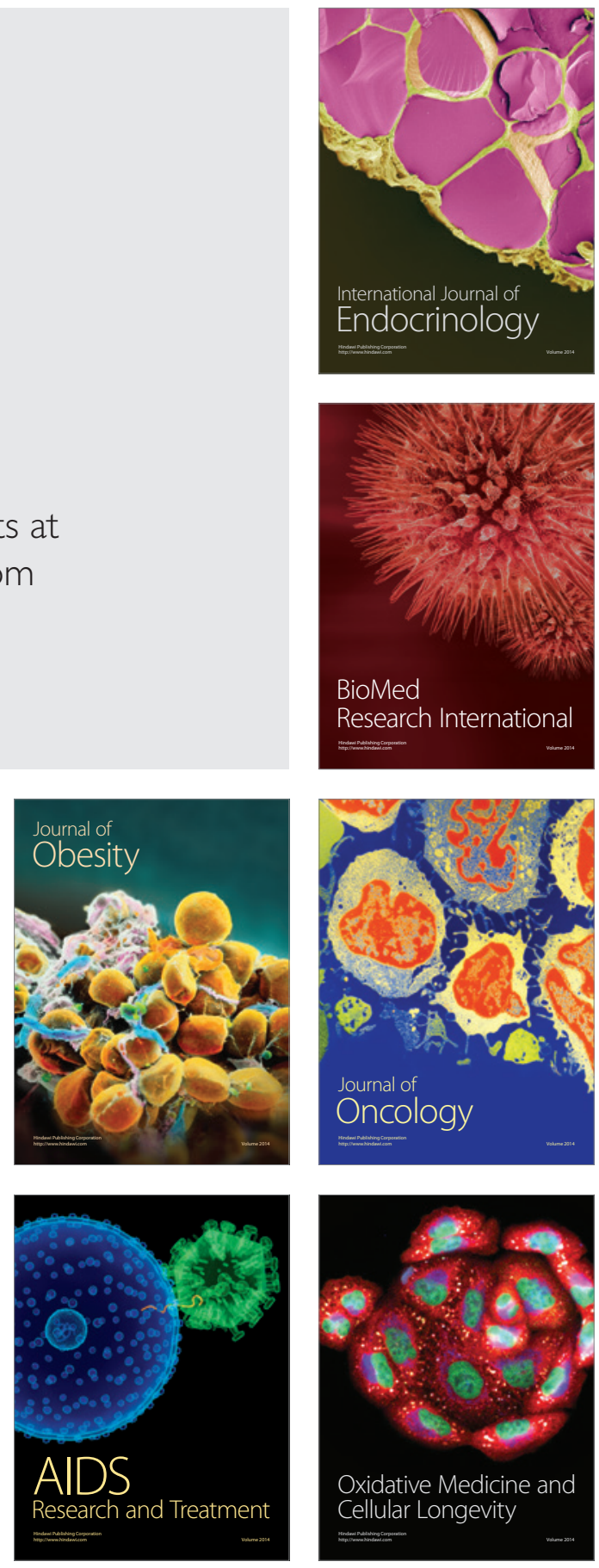